\title{
Optimised spectral kurtosis for bearing diagnostics under electromagnetic interference
}

\author{
Wade A. Smith*, Zhiqi Fan, Zhongxiao Peng, Huaizhong Li and Robert B. Randall \\ School of Mechanical and Manufacturing Engineering, University of New South Wales \\ Sydney, NSW 2052, Australia \\ *Corresponding author. Email: wade.smith@unsw.edu.au \\ Telephone: +6129385 6005, fax: +61296631222
}

\begin{abstract}
The selection of the optimal demodulation frequency band is a significant step in bearing fault diagnosis because it determines whether the fault information can be extracted from the demodulated signal via envelope analysis. Two well-known methods for selecting the demodulation band are the Fast Kurtogram, based on the kurtosis of the filtered time signal, and the Protrugram, which uses the kurtosis of the envelope (amplitude) spectrum. Although these two methods have been successfully applied in many cases, the authors have observed that they may fail in specific environments, such as in the presence of electromagnetic interference (EMI) or other impulsive masking signals.
\end{abstract}

In this paper, a simple spectral kurtosis-based approach is proposed for selecting the best demodulation band to extract bearing fault-related impulsive content from vibration signals contaminated with strong EMI. The method is applied to vibration signals obtained from a planetary gearbox test rig with planet bearings seeded with inner and outer race faults. Results from the Fast Kurtogram and Protrugram methods are also included for comparison. The proposed approach is found to exhibit superior diagnostic performance in the presence of intense EMI.

Another contribution of the paper is to introduce and explain the issue of EMI to the condition monitoring community. The paper outlines the characteristics of EMI arising from widely-used variable frequency drives, and these characteristics are used to simulate an EMI-contaminated vibration signal to further test the performance of the proposed approach. Although EMI has been acknowledged as a serious problem in many industrial cases, there have been very few studies showing its adverse effects on machine diagnostics. It is important for analysts to be able to identify EMI in measured vibration signals, lest it interfere with the analysis undertaken.

Keywords: rolling element bearing; bearing diagnostics; demodulation band; envelope analysis; electromagnetic interference.

\section{Introduction}

Rolling element bearings (REBs) are among the most widely used components in rotating machinery and their failure a common cause of machine breakdown [1]. Such failures can be catastrophic or lead to the shutdown of entire production lines, potentially causing personal damage or economic loss [2], so it is essential to accurately detect and diagnose bearing faults at an early 
stage.

Envelope analysis has been widely applied in bearing diagnostics for extracting the fault information. This is because the impulses caused by bearing faults usually excite resonances at much higher frequencies than the vibration generated by other machinery components, and this resonant response is considered to be amplitude modulated at the characteristic defect frequency [3]. Nevertheless, envelope analysis has a major challenge in the selection of the most suitable band for amplitude demodulation.

The band selection issue has largely been solved using spectral kurtosis (SK), a statistical tool which indicates the impulsivity of a signal as a function of frequency. Being sensitive to the non-stationary parts of the vibration signal, SK can reveal the frequency and bandwidth of the resonance(s) excited by the bearing fault and thus the most promising frequency band that should be used for demodulation and subsequent envelope analysis. A number of studies relating to SK and its practical implementation have been presented over the last decade. Antoni [4] gave a comprehensive theoretical framework of SK and presented an SK estimation method based on the short-time Fourier transform (STFT). Antoni and Randall [5] published a further study on how to apply SK in the diagnostics of rotating machinery, and they presented a tool called the Kurtogram, which shows SK values in a special band-pass structure as a function of two parameters, centre frequency and bandwidth of the filtered signal. The Kurtogram determines the optimal frequency band for demodulation by choosing parameters that maximise the SK value. Considering that it is time-consuming to compute the Kurtogram with all possible combinations of centre frequencies and bandwidths, the Fast Kurtogram was introduced by Antoni [6]. This method applies a 1/3-binary tree Kurtogram, which means it only investigates SK values in a few given bandwidths and greatly reduces the computing time.

A number of improved Kurtogram-based methods have since been proposed by other researchers. Sawalhi et al. [7] developed a Wavelet Kurtogram, based on complex Morlet wavelets. They argued that wavelets are well-suited to such an application because they have uniform resolution on a logarithmic frequency scale, which corresponds to impulse responses with a constant value of damping ratio. Lei et al. [8] adopted the wavelet packet transform (WPT) as the filter for the Kurtogram, with the purpose of ovecoming the drawbacks of STFT or FIR filters.

Arguing that the temporal-based kurtosis indicator of the Kurtogram performs poorly in the presence of strong non-Gaussian noise, Barszcz and Jabłoński [9] proposed a method called the Protrugram, which employs the kurtosis of the envelope (amplitude) spectrum rather than of the envelope time signal. A similar fault indicator was used in [10], in which the WPT-based Kurtogram was optimised by calculating the kurtosis of the envelope power spectrum.

In recent laboratory testing on a planetary gearbox test rig, the authors encountered a situation in which the Fast Kurtogram and Protrugram approaches failed to yield successful diagnoses of large seeded REB faults. Inspection of the measured signals revealed a form of impulsive wideband interference, which later investigations determined was in fact electromagnetic interference (EMI) 
arising from the variable frequency drive (VFD) controlling the induction motor of the test rig. It became clear that this interference was adversely affecting the diagnostics, rendering these established techniques incapable of diagnosing even large seeded faults. Since these tests, the authors have encountered a number of other instances of EMI contamination in vibration signals, most often, but not always, arising from VFDs (see Section 2).

This experience prompted the development of a simple SK-based approach to demodulation band selection, which the authors refer to as Optimised Spectral Kurtosis. Though applied here to vibration signals contaminated with EMI specifically from variable frequency drives, it is thought the approach will have broader applicability to other cases involving impulsive wideband ${ }^{1}$ interference.

The remainder of this paper is organised as follows. Section 2 gives a brief explanation of the nature of the interference from VFDs. This is by no means an exhaustive treatise, but is rather intended to outline the most common type of EMI from VFDs, that arising from the pulse-width modulated (PWM) control signal used by VFDs. Section 3 provides a basic review of the Fast Kurtogram and Protrugram, and then introduces the Optimised SK approach for demodulation band selection. Section 4 explains the experimental and simulated data used in this study, and the diagnostic performance of the three methods - Fast Kurtogram, Protrugram and Optimised SK - is illustrated in Section 5. The discussion in Section 6 includes a comparison of the three methods, and conclusions are given in Section 7.

\section{Electromagnetic interference from variable frequency drives}

Electromagnetic interference is defined as any unwanted signal that is either radiated or conducted to electronic equipment and negatively affects the performance of the equipment [11]. The present focus is on the contamination of vibration signals with EMI generated from variable frequency drives. From the authors' experience, this type of EMI is very common. Indeed, VFDs are widely employed in industrial applications for controlling AC motor speed and torque by altering motor input frequency and voltage, and EMI is commonly associated with VFDs [12].

\subsection{Pulse-width modulated signals}

As explained by Detrich et al. [13], when used with an induction motor, a VFD converts an AC voltage signal to an AC output of a different voltage and frequency. VFDs consist of three sub-systems: a rectifier to convert the AC input to a DC signal, a DC-link, and an inverter to convert back to an AC signal, which is then supplied to the motor. A number of VFD topologies exist, but by far the most common are voltage-source inverter drives with pulse-width modulated (PWM) output signals [14]. These PWM signals can be generated by comparing a reference signal $r(t)-$ an AC signal of the required frequency $f_{r}$ to obtain the correct motor speed - with a carrier signal $c(t)$, as follows [15]:

\footnotetext{
1 The term 'wideband' is used here to describe a signal occupying a wide frequency range, regardless of its spectral form (such as its shape and whether it is dominated by discrete or random components); this is as opposed to 'broadband', which the authors believe implies a signal with a smooth, flat spectrum.
} 


$$
X_{P W M}(t)=\operatorname{sgn}[r(t)-c(t)]
$$

The carrier signal is generally a sawtooth or triangle waveform, and the carrier frequency $f_{c}$ called the switching frequency. The switching frequency of a VFD can often be set by the user, and commonly falls in the range $2-15 \mathrm{kHz}$ [13]. The frequency $f_{r}$ of the reference signal has been called the 'pseudo line frequency' [13], and this terminology is adopted here. When integrated, a PWM signal creates an approximate sinusoid at the pseudo line frequency, but with higher frequency content. Fig. 1 shows an example of this process, where the effect of switching frequency on the generated sinusoid is clearly visible.

As outlined by Sun [15], the Fourier analysis of PWM signals is quite complex, and certainly beyond the scope of this paper, but the spectra of PWM waveforms are known to include the following discrete components:

1. Harmonics of the pseudo line frequency $f_{r}$

2. The carrier (switching) frequency and its harmonics, at $f=n f_{c}, n=1,2, \ldots, \infty$

3. Sidebands spaced at pseudo line frequency around the carrier harmonics, at $f=n f_{c} \pm m f_{r}, m=1,2, \ldots, \infty$, with distribution dependent on the precise nature of modulation (i.e., the nature of the sawtooth/triangle waveform, which can produce trailing-, leading- or double-edge modulation) [15]

In the case shown in Fig. 1 (trailing-edge modulation), $f_{c} / f_{r}=10$, but in practice a much larger ratio would be used, leading to a smoother generated sinusoid but with the same spectral characteristics. 
$\mathrm{a}$

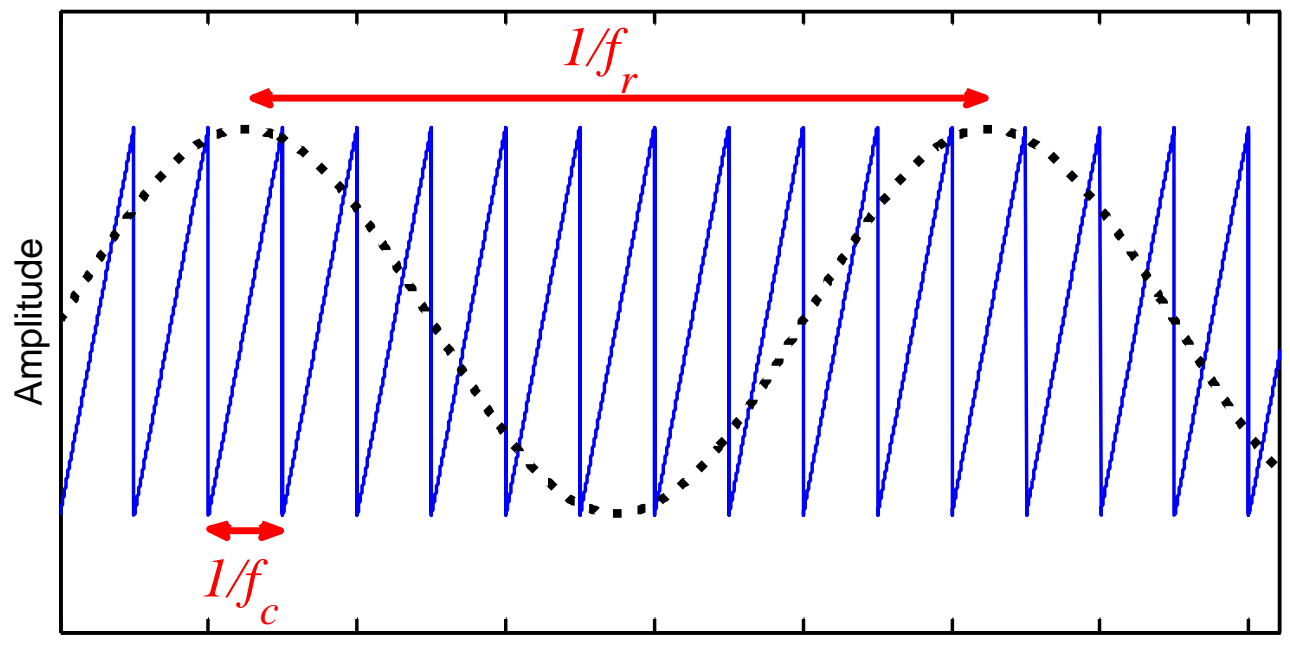

b

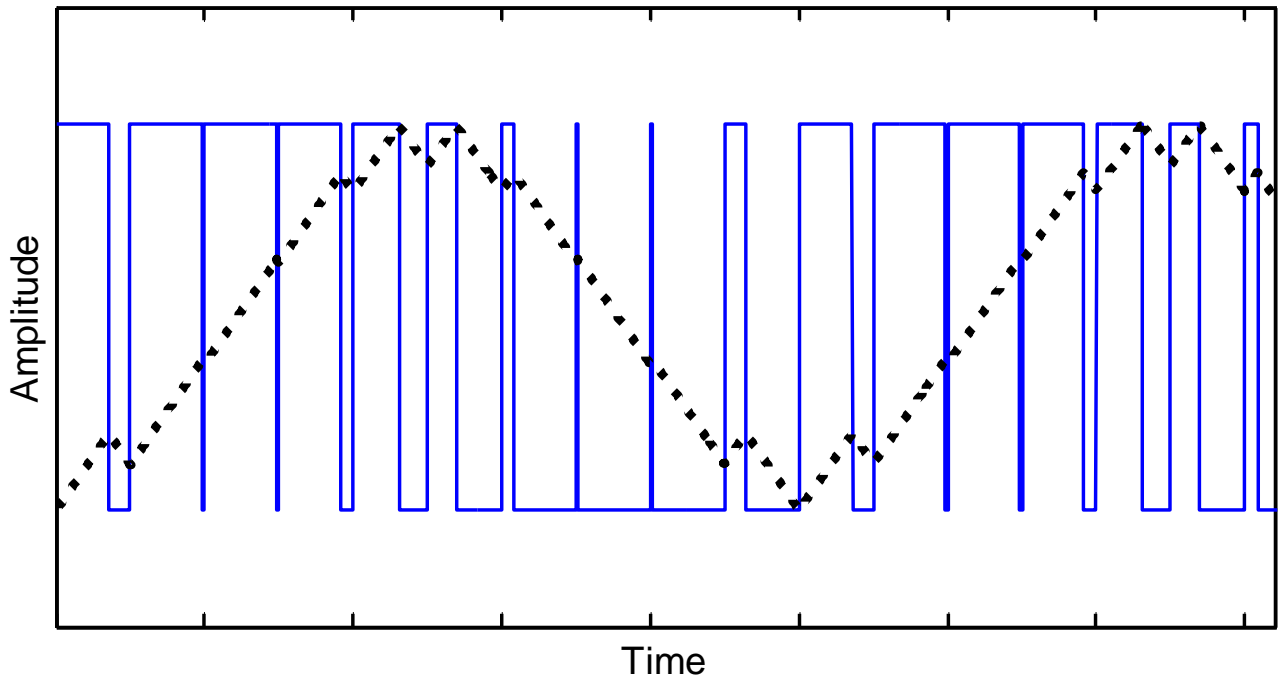

Fig. 1 Generation of apparent sinusoid from pulse-width modulated signal; (a) reference $(\cdots)$ and carrier (-) signals; (b) pulse-width modulated signal (-) and generated sinusoid ( $\cdots$ )

\subsection{Measured electromagnetic interference from a variable frequency drive}

In recent testing on a gearbox test rig (see Section 4.1.1), the authors obtained acceleration signals with the same spectral characteristics as described above. An example is shown in Fig. 2, which displays the power spectral density (PSD) and the zoomed spectrum of a signal recorded with the VFD switching frequency set at $14 \mathrm{kHz}$ and a pseudo line frequency of $24 \mathrm{~Hz}$. It can clearly be seen that the switching frequency and its harmonics dominate the spectrum above about $12 \mathrm{kHz}$. A spectral plot zoomed around the second harmonic of the switching frequency is shown in Fig. 2(b) to illustrate the sideband structures. This shows that the sideband spacing around $f_{c}$ corresponds not only to the pseudo line frequency of $24 \mathrm{~Hz}$, but also to $100 \mathrm{~Hz}$, which is twice mains frequency ${ }^{2}$. To confirm this interference was in fact EMI from the VFD, the authors conducted a number of further tests with a range of carrier and pseudo line frequency settings, and similar characteristics were found in all the obtained signals: harmonics of the carrier frequency surrounded by sidebands spaced at both the pseudo line and mains frequencies.

2 The term 'mains frequency' is used here to describe the frequency supplied by the grid, which is $50 \mathrm{~Hz}$ in Australia. 
$\mathrm{a}$

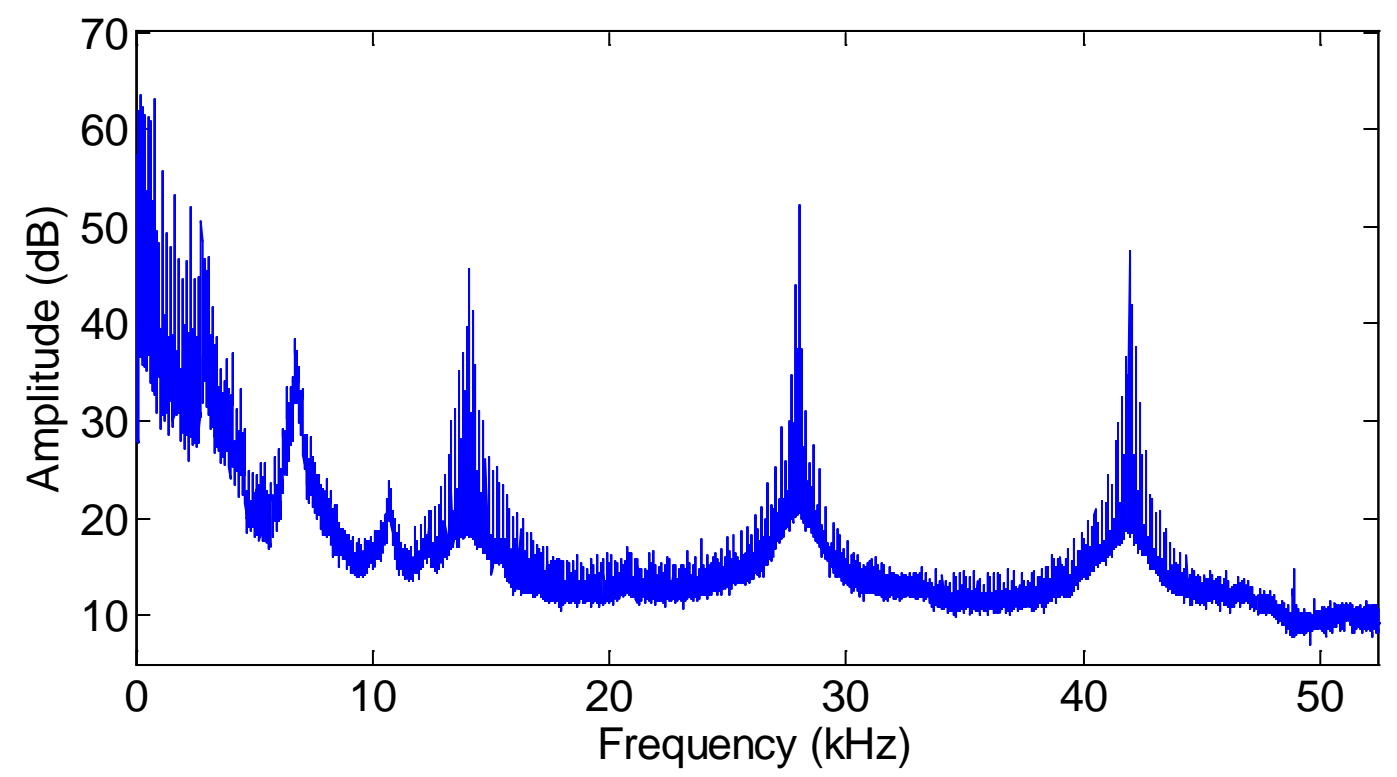

b

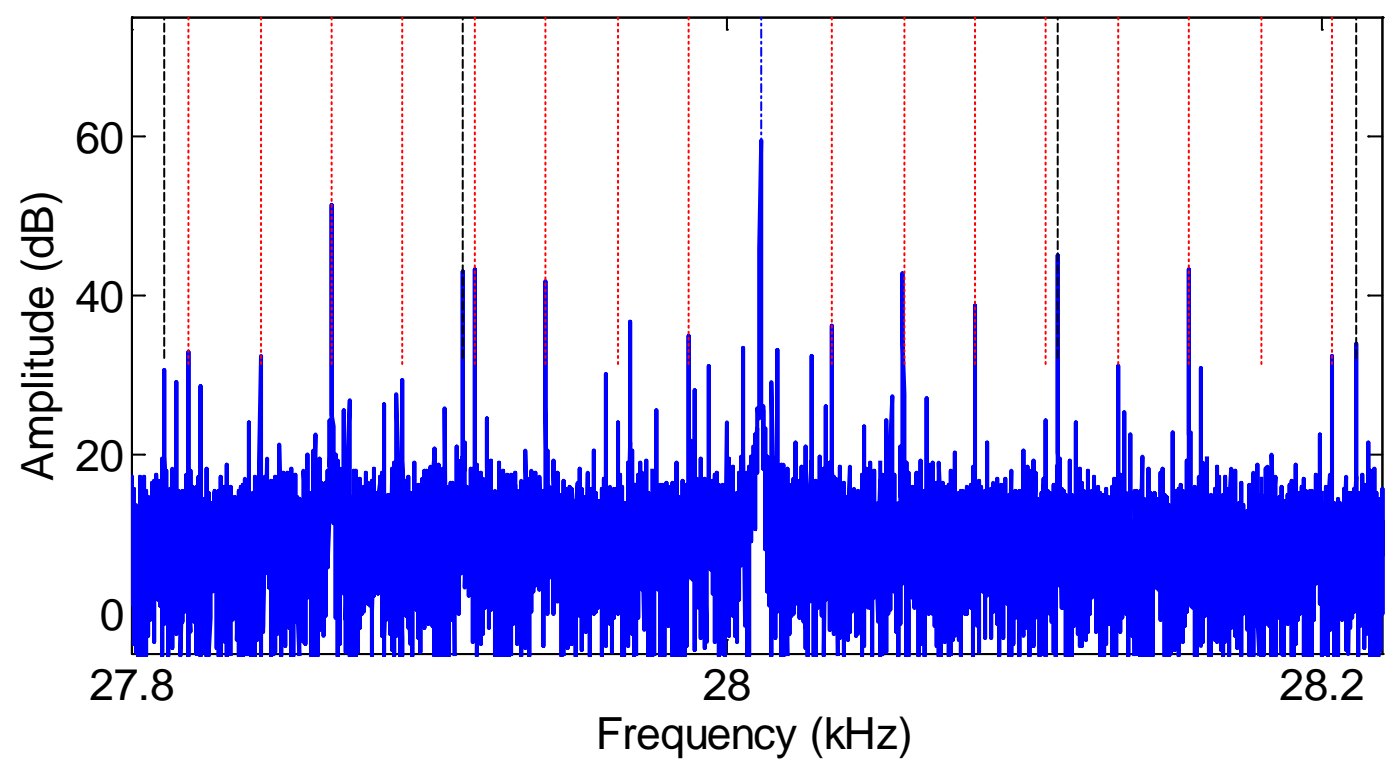

Fig. 2 Acceleration signal dominated by EMI; (a) PSD; (b) zoomed spectrum around $28 \mathrm{kHz}$ (second harmonic of carrier frequency); data cursors spaced at $24 \mathrm{~Hz}$ (red dot) and $100 \mathrm{~Hz}$ (black dash) around carrier (blue dash-dot)

Later, the time waveforms of the measured signals were closely inspected. This inspection revealed that the signals had a number of seemingly random 'dropouts', sharp spikes spanning perhaps two or three samples' duration, each immediately preceded and followed by a sawtooth oscillation. An example of two dropouts is shown in Fig. 3. These dropouts were thought at first to be unrelated to the observed EMI, but it is now understood that this is a common manifestation of EMI from PWM signals. 


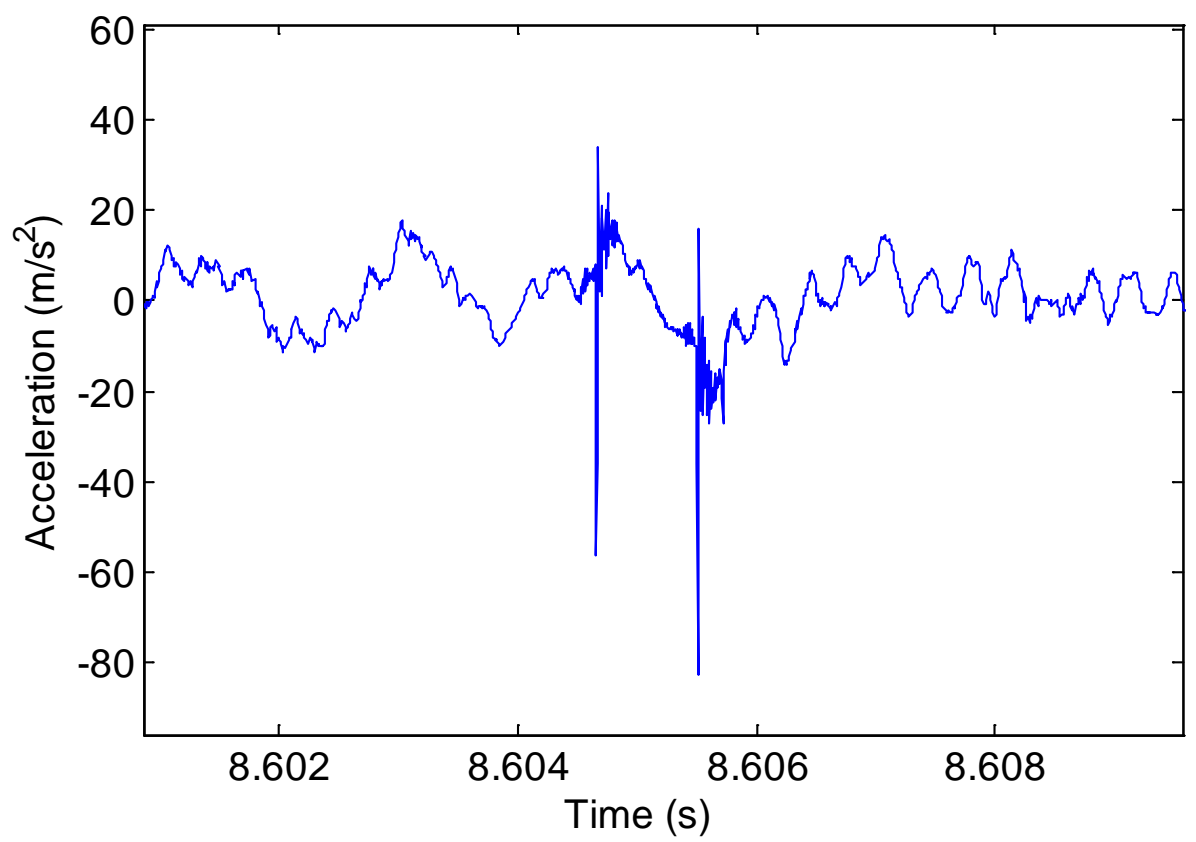

Fig. 3 Acceleration signal dominated by EMI; time waveform showing ‘dropout’ effect

Skibinski et al. [11] explain that EMI from PWM-based variable frequency drives can be generated from a number of different mechanisms, the major one being large line-to-ground current spikes. In such cases, a very high frequency transient is generated with each rise of the PWM voltage; each fall in the PWM signal also generates a transient, but with the opposite sign. This concept is outlined in Fig. 4. Note that in general the frequency of the transient would be very high compared with typical sampling rates used in condition monitoring.

It was thus concluded that the observed dropouts in the measured signals are in fact transients stemming from very high frequency current (and voltage) oscillations, which when passed through a typical anti-aliasing filter can appear as two- or three-sample dropouts preceded and followed by sawtooth oscillations (at about 0.4 times the sampling rate) reflecting the response of the anti-aliasing filter. It was observed in the measured signals that dropouts occurred only in the negative direction, were quite random in magnitude, and in fact were not apparent at every rise or fall point in the PWM signal. This is reflected in Fig. 3, where there are two large dropouts but no others (in the period shown). It is thought that in many such cases smaller dropouts still occur but are buried in noise.

Because the dropouts are often indistinguishable from noise, and because they have non-constant spacing (from modulation in the PWM signal), they were at first thought to be unrelated to EMI. The connection to EMI was only made after writing an automated program that identified the dropouts, replaced each one with a delta function, and then conducted Fourier analysis of the result. This revealed the spectral form described in Section 2.1 (switching frequency harmonics surrounded by sidebands spaced at the pseudo line frequency), and thus confirmed the connection.

Clearly, this interference in the form of a series of transients with a complex modulation structure would be very problematic for bearing diagnostics, since it mirrors precisely the features sought in 
most diagnostic methods: impulsivity and periodicity. Furthermore, the wideband nature of the interference is likely to span the typical frequency range at which bearing faults often manifest themselves, and the EMI modulation frequencies - the pseudo line frequency and its first few harmonics - are likely to span the typical bearing fault frequency range.

$\mathrm{a}$

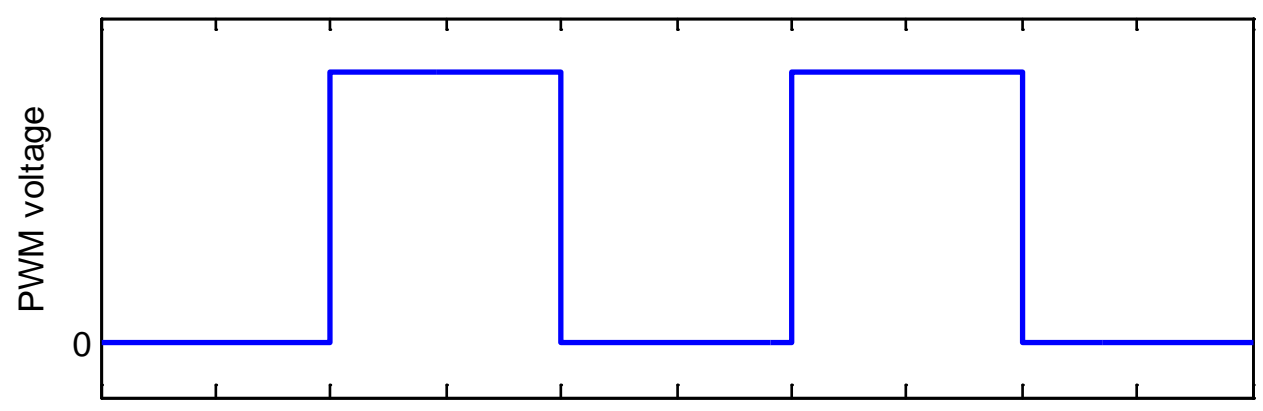

b

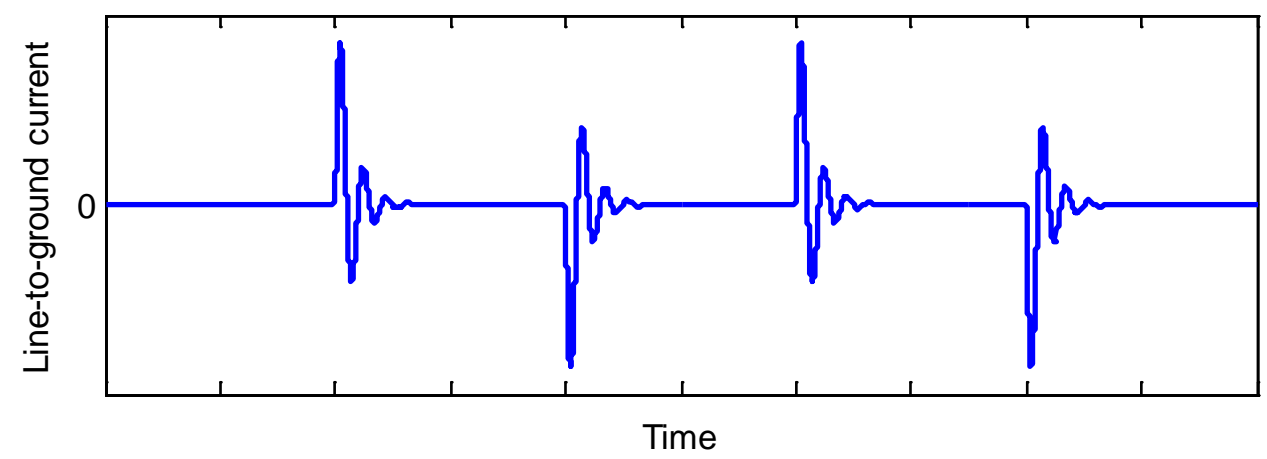

Fig. 4 EMI manifestation from PWM signal; (a) PWM voltage; (b) induced line-to-ground current transients

\subsection{Electromagnetic interference and bearing diagnostics}

The EMI generated from VFDs has been found to be a serious problem which can affect transducers' ability to record desired signals and adversely affect the operation of other electronic devices or systems [16-18]. The characteristics of VFD-generated electromagnetic interference have been detailed in the literature [11, 12, 19, 20], yet there appear to have been very few studies specific to machine condition monitoring and how EMI may affect the detection and diagnosis of faults in machinery.

This topic was touched upon by Detrich et al. [13], who outlined how the EMI from VFDs could affect measured vibration signals, and they analysed the frequency domain distribution of EMI in recorded acceleration signals. Quezada Acuña and Molina Vicuña [21] recently reported on the problem of EMI in acoustic emission signals, and how it interfered with bearing diagnostics using conventional methods. To overcome this, they proposed a method called the 'Cyclostationary Peak Ratio Distribution' based on the strength of components at specific cyclic frequencies (bearing fault frequencies) in the spectral coherence map. However, the method requires the generation of a separate bandpass filter for each cyclic frequency under investigation, so its usefulness in practice may be limited.

Smith et al. [22] found that EMI severely interfered with planet bearing diagnostics when studying 
data from the same test rig as in the present study (see Section 4.1.1). The presence of EMI strongly affected the performance of the Fast Kurtogram, and the wrong demodulation band with no bearing fault information was selected in many of the conducted analyses [22].

Since these tests, the authors have encountered a number of other instances of EMI contamination in vibration signals, most often, but not always, arising from VFDs. Indeed, two of the authors recently documented how much of the widely-used Case Western Reserve University bearing data is contaminated with EMI from a dynamometer controller [23]. Other potential sources of EMI are power transmission lines, switched-mode power supplies and household electrical appliances. The authors believe that EMI contamination of vibration signals - albeit not usually to the extent of that shown in Fig. 2 - is a more common issue than is readily acknowledged. The structured, wideband and impulsive nature of the inteference, coupled with the fact that bearing faults often manifest themselves as impulse responses of high frequency and relatively low energy, suggests that bearing diagnostics would be particularly vulnerable to EMI.

In view of these issues, this paper presents a simple method for determining an optimal demodulation band for bearing diagnostics under electromagnetic interference. This Optimised SK approach is based on the traditional SK, but it borrows from the Protrugram [9] the idea of setting the demodulation bandwidth to a fixed minimum value based on the known bearing parameters, and then searching for the optimal centre frequency. Because of this characteristic, even though it is applied here to vibration signals contaminated with EMI specifically from a VFD, it is thought the basic idea will have broader applicability to other cases of impulsive wideband interference.

\subsection{Filtering of electromagnetic interference from vibration signals}

Initial inspection of the measured signals outlined in Section 2.2 suggested the EMI problem was essentially described by discrete frequency components (harmonics of the VFD switching frequency surrounded by sidebands), so it was decided to apply some sort of discrete/random separation technique to remove the interference. A number of such filtering attempts were made on these signals, including DRS [24], cepstrum prewhitening [25] and cepstrum editing [26], but none proved very successful in removing the interference.

An example of such filtering is displayed in Fig. 5, which shows a cepstrum editing plot of a measured acceleration signal obtained with a switching frequency of $14 \mathrm{kHz}$ and a pseudo line frequency of $24 \mathrm{~Hz}$. The plot has been zoomed to highlight the EMI components around the switching frequency, which are very clear in the raw signal. A relatively new cepstrum editing technique [26], designed to remove specific harmonics and sideband families, was applied to obtain the filtered signal in the figure. It appears from the figure that the filtering was very successful, with the EMI components almost entirely indistinguishable from background noise. However, after demodulating a band around the switching frequency (centre frequency $C F=14,000 \mathrm{~Hz}$, bandwidth $B W=1,024 \mathrm{~Hz}$ ) and forming the squared envelope spectra (Fig. 6(a) and Fig. 6(b) for the raw and filtered signals, respectively), it is clear that EMI components are still dominant. This shows that it is not only the large-amplitude discrete components of the EMI that are problematic, but also its phase properties. This can be understood with reference to the dropouts observed in the time records 
(Section 2.2), which were only recognised after the filtering attempts were made. These dropouts are virtually delta functions, which are represented by a very broad spectrum comprising a large number of components all lining up in phase at the same time.

That is, the interference is both first- and second-order cyclostationary (CS1 and CS2). While the CS1 components are easily removed with the conventional time-invariant filtering methods attempted here, the CS2 components remain. These second-order components could perhaps be filtered out using time-variant filtering [27], but this has not been explored further.

Since these filtering attempts failed to remove the interference from the measured signals, the studied diagnostic techniques (see Section 3) were applied to the raw signals, without any preprocessing.

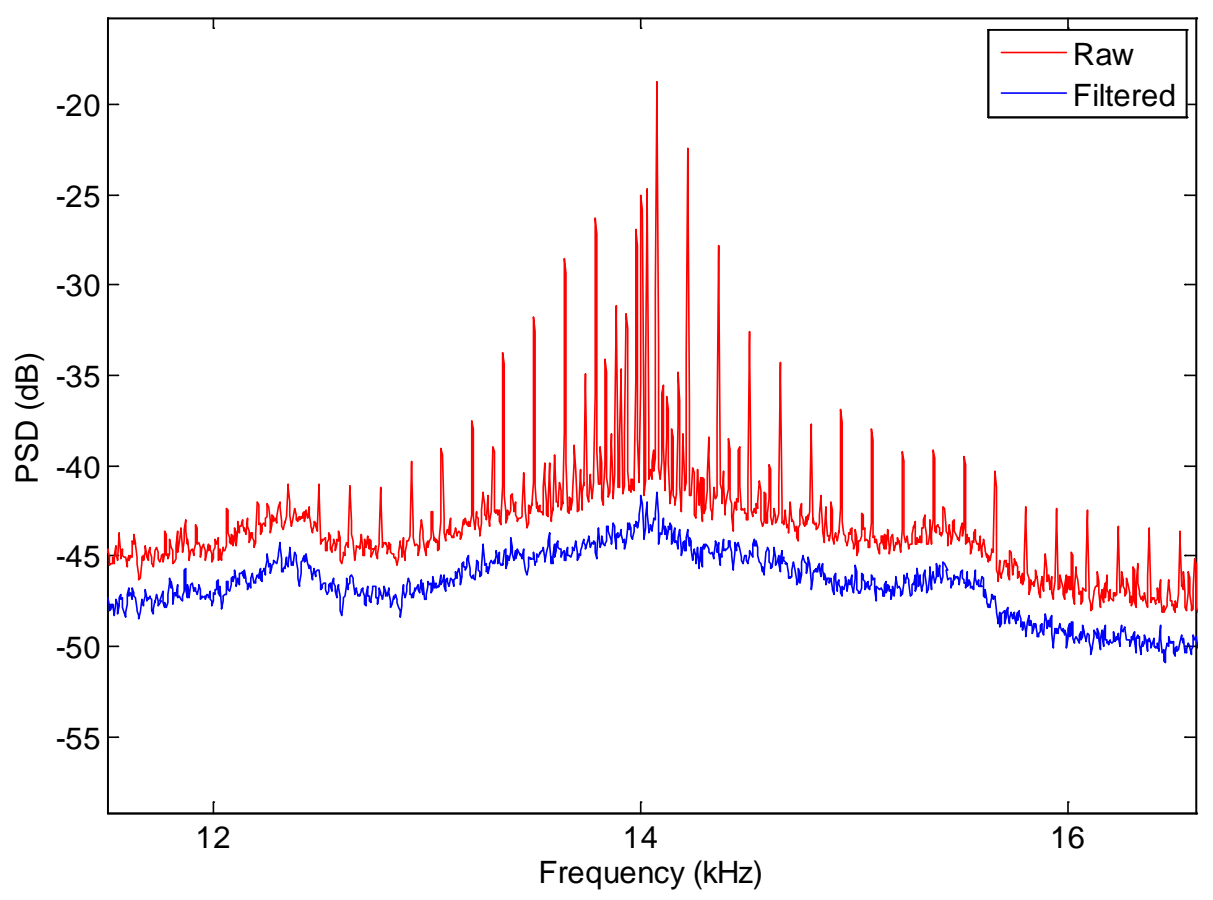

Fig. 5 Comparison of cepstrum editing of raw signal and filtered signal (zoomed around $14 \mathrm{kHz}$ ) 


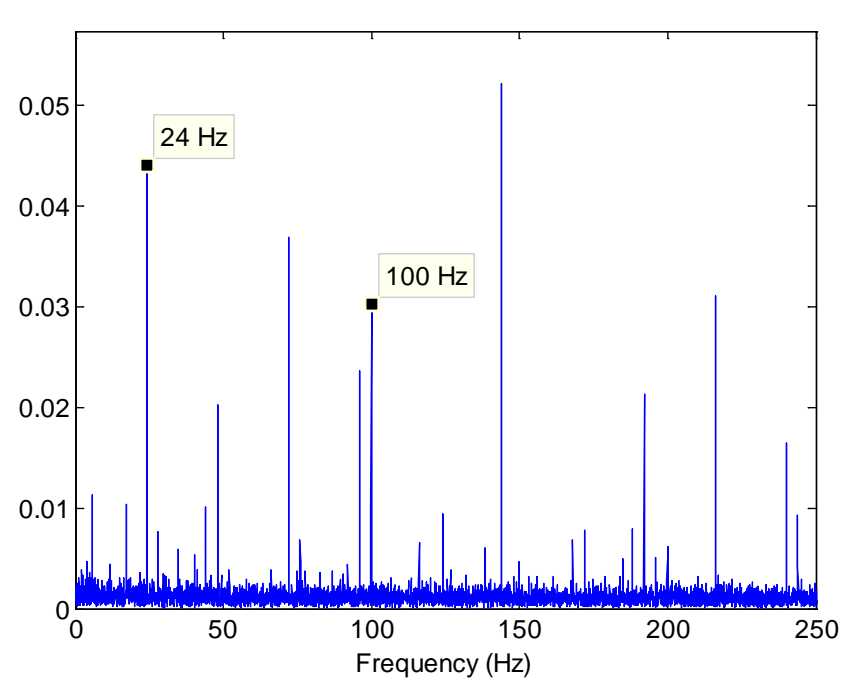

b

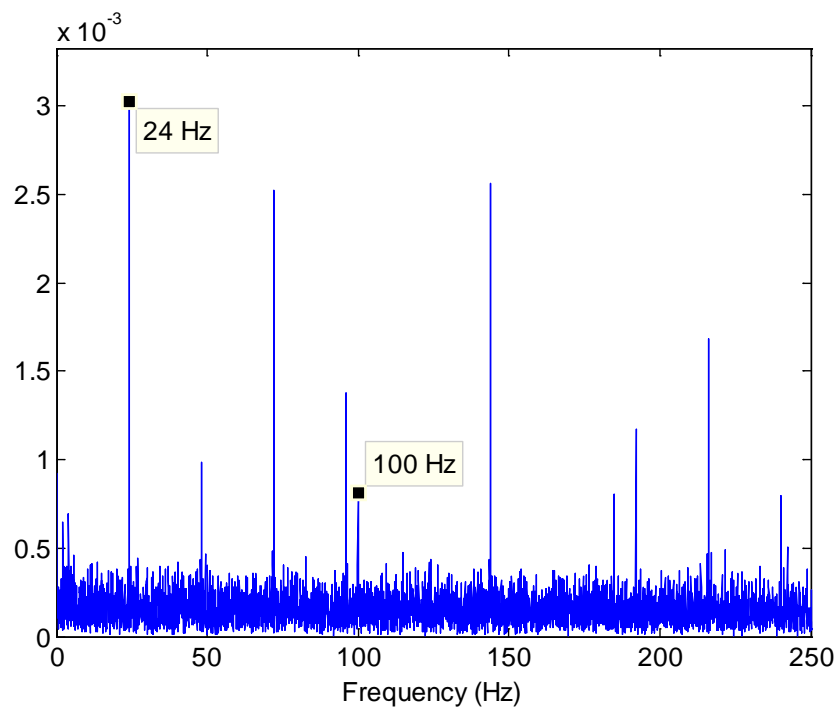

Fig. 6 Squared envelope spectrum $(C F=14,000 \mathrm{~Hz}, B W=1,024 \mathrm{~Hz})$; (a) raw signal; (b) filtered signal

\section{Applied demodulation band selection methods}

Further details of the Fast Kurtogram and Protrugram techniques are reviewed in this section, before the proposed Optimised SK approach is introduced and explained.

\subsection{Review of the Fast Kurtogram}

The Fast Kurtogram was first proposed in [6] as an efficient algorithm for detecting the existence of non-stationarity in signals and showing its location in the frequency domain. This method is based on spectral kurtosis, which is a fourth-order statistical tool and is very sensitive to impulsive signals. SK was estimated based on the short-time Fourier transform (STFT) in [4], and can be expressed as follows:

$$
K(f)=\left\langle|X(t, f)|^{4}\right\rangle /\left\langle|X(t, f)|^{2}\right\rangle^{2}-2
$$

Where $X(t, f)$ is the (complex) time/frequency envelope of the signal, and the square of its amplitude gives the power spectrum at each time $t .\langle$.$\rangle is the time-averaging operator.$

Because the SK value varies with both the frequency $f$ and frequency resolution $\Delta f$, a representation of SK in the plane $(f, \Delta f)$ is convenient for finding the maximum value of SK. However, the full exploration of SK over the entire $(f, \Delta f)$ plane - the full Kurtogram - is too time-consuming to apply in most industrial applications [6]. The Fast Kurtogram solves this issue by applying a 1/3-binary tree structure for designing band-pass filters and investigating the SK value of the filtered signal from selected bandwidths. The Fast Kurtogram comprises a 2D plane in which the horizontal axis represents the frequency and vertical axis represents the frequency resolution. A colour scale shows SK values for each frequency band. The band (part of the Kurtogram grid) with the maximum SK value is then chosen as the optimal demodulation band for subsequent envelope analysis. 
Although the Fast Kurtogram has proven to be effective in many cases [1, 28], including in the presence of strong Gaussian noise, it has been found to be ineffective in several conditions, as illustrated in [8-10, 29]. The performance of the Fast Kurtogram may be poor when the signal-to-noise ratio is relatively low, or strong non-Gaussian noise exists [9]. The latter condition describes the EMI contamination studied here, for which the Fast Kurtogram was found to be unsuccessful in a number of cases.

\subsection{Review of the Protrugram}

Intending to overcome the drawbacks of the Fast Kurtogram, the Protrugram [9] was developed based on the kurtosis of the envelope (amplitude) spectrum rather than of the envelope time signal. Another feature of the Protrugram is the setting of the bandwidth (frequency resolution) to a fixed (usually minimum) value, based on the mechanical parameters of the monitored machine, such as the bearing characteristic defect frequencies for bearing diagnosis. The Protrugram is therefore not a blind technique (unlike the Fast Kurtogram), since knowledge of the system parameters is required. The selected bandwidth should include at least the first three harmonics of the characteristic frequencies of the bearing(s) under study [9]. Too narrow a bandwidth (less than the fundamental sought frequency) will fail in reflecting the fault information in the envelope spectrum. If the bandwidth is too wide, more noise will be included in the filtered signal and the bearing fault information may be difficult to extract from the noise.

After the bandwidth is chosen, the optimal demodulation band is determined by stepping through the candidate centre frequencies (across the available frequency range), generating the envelope spectrum for each and calculating its kurtosis, and then selecting the band returning the highest kurtosis value. As noted in [9], the selection of centre frequency step size involves a trade-off between computation time and accuracy (frequency resolution), and Barszcz and Jabłoński found that a step size of about $100 \mathrm{~Hz}$ was a reasonable choice in their application.

This capability to finely select the optimal centre frequency is a great advantage of the Protrugram over the Fast Kurtogram, a feature that prompted Zhang and Randall [2], in an earlier study, to propose the augmentation of the Fast Kurtogram with genetic algorithms designed to fine-tune the centre frequency selection.

Yet despite this apparent advantage, the Protrugram was found here to be unsuccessful in diagnosing bearing faults in the presence of strong EMI.

\subsection{Proposed method: Optimised Spectral Kurtosis}

The poor diagnostic performance of these established methods led the authors to optimise the traditional spectral kurtosis to achieve an improved band selection process. The method was first applied in [30], but without detailed explanation and analysis. It was found here to select correct fault-related demodulation bands even though the vibration signals were strongly masked by EMI. The method is thought to be sufficiently general to apply to any situation where impulsive wideband masking signals are present, where the Kurtogram and Protrugram may prove unsuccessful. 
A flow chart showing the implementation of the Optimised SK approach is shown in Fig. 7. The approach provides a representation of the traditional SK calculated with the same parameter requirements as the Protrugram, with the demodulation bandwidth pre-set. Based on the suggestions in [9], it is recommended here to set the bandwidth as narrow as possible, but to include at least the first three harmonics of the bearing fault frequency. The idea is that because the EMI occupies such a wide frequency range (several kilohertz), minimising the bandwidth maximises the signal-to-noise ratio. This may not be correct if the bandwidth of the resonance(s) excited by the bearing fault signal is itself very broad, but even in such cases, the use of a narrow bandwidth gives the possibility of extracting those parts of broad resonances least contaminated by interference.

Next, the optimal centre frequency is investigated using a stepping process. The centre frequency $C F$ is shifted through a predefined step $s p$ in the range $\left[B W / 2, f_{s} / 2-B W / 2\right]$, where $f_{s}$ represents the sampling frequency. For each $C F$, the signal is bandpass filtered over the range $[C F-B W / 2$, $C F+B W / 2$ ], and one side of its spectrum is then transformed back to the time domain to obtain the envelope, the kurtosis of which is then calculated and stored. After calculating the kurtosis values for every $C F$ in the range, the optimal centre frequency is chosen as that with the maximum kurtosis.

The overall architecture of the approach is identical to the Protrugram, but in fact the use of the kurtosis of the envelope time signal means the result is simply the traditional spectral kurtosis calculated using the best possible bandwidth settings for the application. The process could thus be described as looking along one row of the (full) Kurtogram, differing importantly from the Fast Kurtogram in that the step size for determining successive $C F$ candidates can be set as desired and is not limited by the bandwidth. 


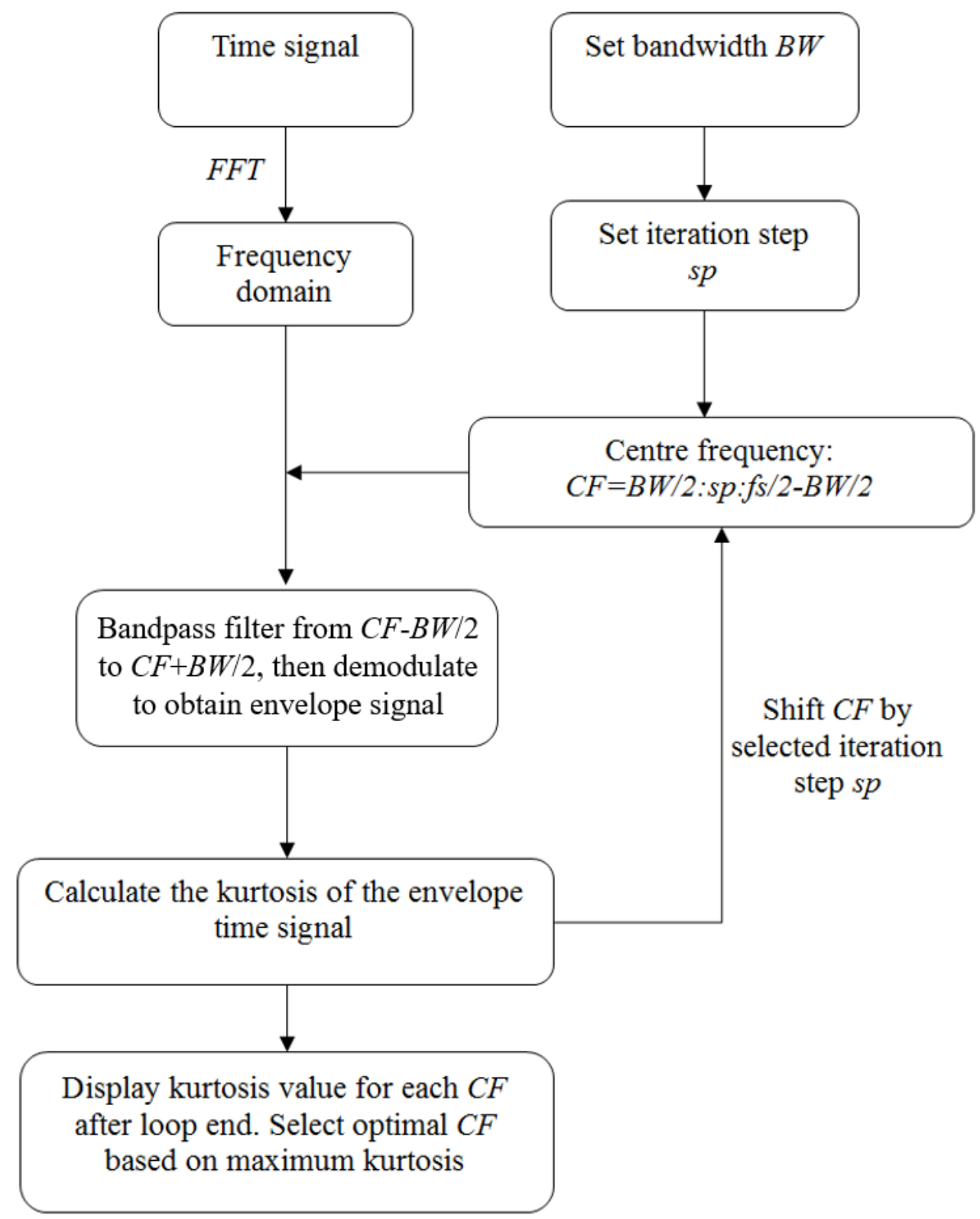

Fig. 7 Flow chart of the proposed method

\section{Description of analysed vibration signals}

\subsection{Experimental data}

\subsubsection{Test equipment}

The planetary gearbox test rig used in this study is pictured in Fig. 8. A three-phase induction motor drives a hydraulic system to provide a resistance torque to the gearbox. A torque transducer is employed to measure the applied torque on the gear set. The speed of the driving shaft can be controlled by a VFD (Fuji Electric FRENIC-MEGA model number FRN7.5G1E-4A). 


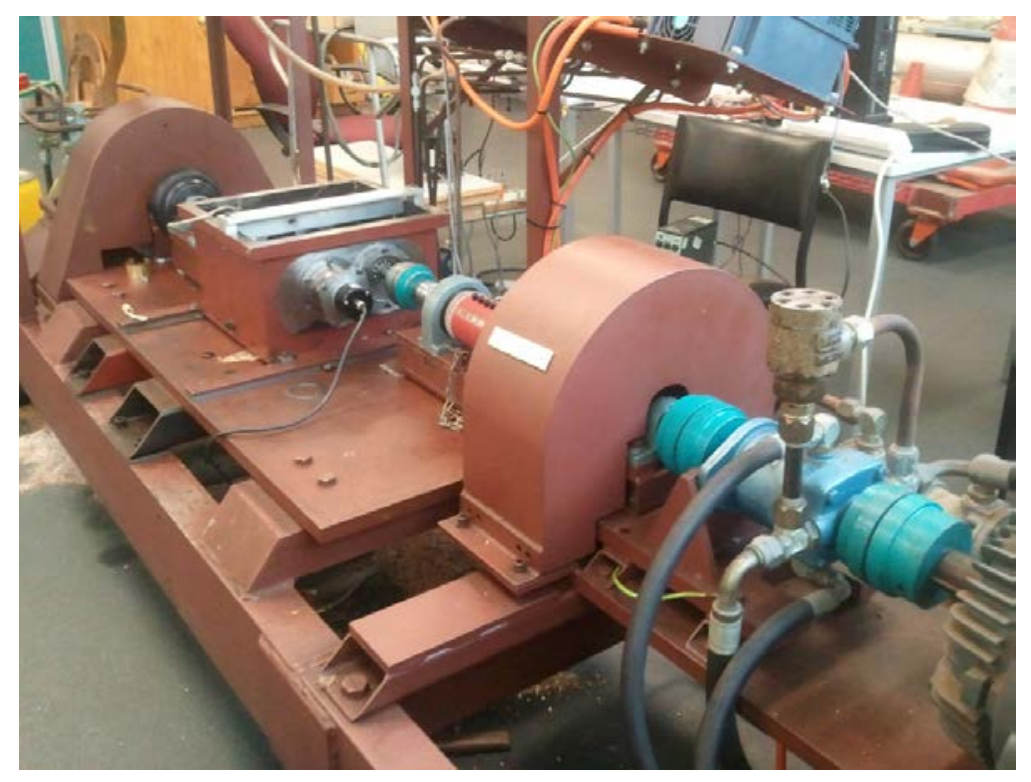

Fig. 8 Planetary gearbox test rig

A schematic of the planetary gearbox is shown in Fig. 9. In the planetary stage, the gear ratio is 1:3 (speed up) with the arrangements of a 40 tooth sun gear, three 20 tooth planetary gears and an 80 tooth ring gear. The ring gear is fixed, while the planet carrier acts as the input to the planetary stage and the sun gear the output. With an initial 90:32 reduction stage, the overall transmission ratio of the test rig is approximately $1: 1$.

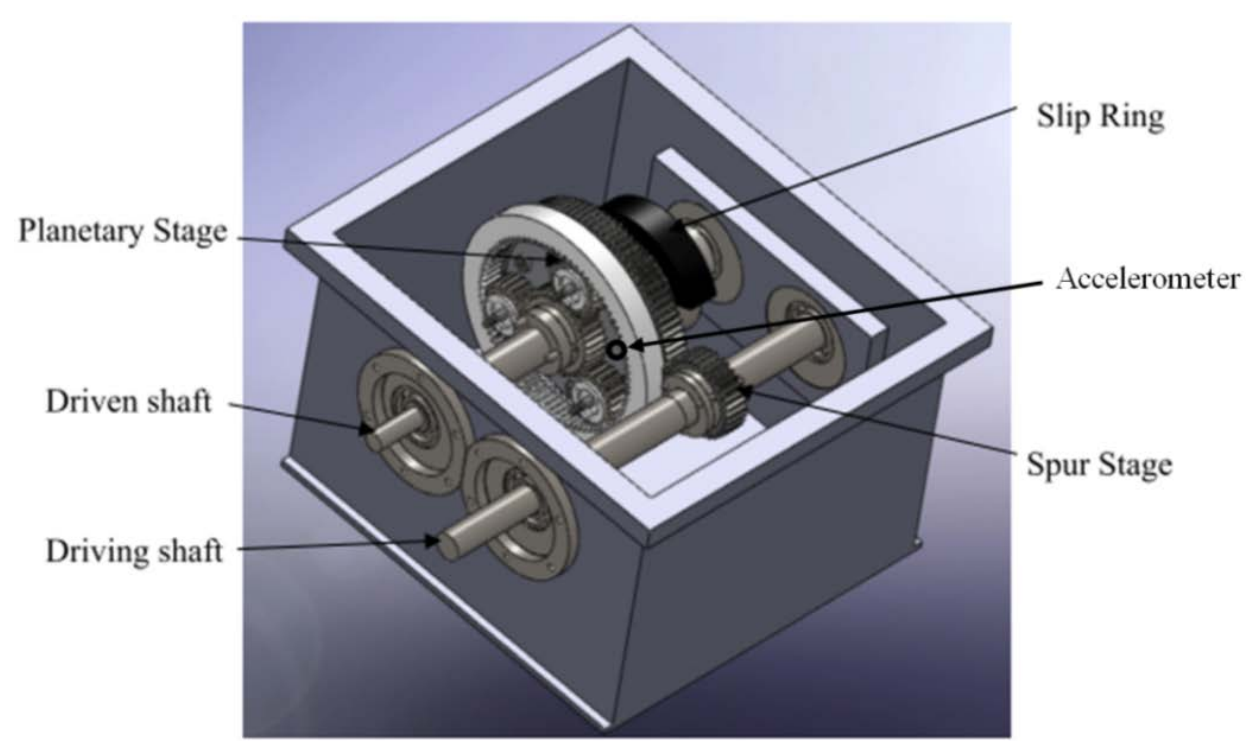

Fig. 9 CAD model of the planetary gearbox

A Brüel \& Kjær 4394 IEPE-type accelerometer was used for vibration measurements. The accelerometer was mounted on the planet carrier in the axial direction, as part of a study to investigate the potential advantages of internal vibration measurements [22, 31]. Since the accelerometer rotates with the planet carrier, a Michigan Scientific B6-2 slip ring was used for transmitting the vibration signal to the signal conditioner. A Brüel \& Kjær PULSE analyser was connected with a laptop running Brüel \& Kjær Labshop software for data recording. The recorded 
data was then exported to MATLAB for further processing.

Faults were seeded in both the inner and outer races of the planet gear bearings. IKO NAF 122413 needle roller bearings were used, each containing 15 rollers of $2 \mathrm{~mm}$ diameter, and the bearing pitch diameter was $18 \mathrm{~mm}$. Electric spark erosion was used for seeding the faults, which were of depth $0.4 \mathrm{~mm}$ and of widths 1.2 and $1.0 \mathrm{~mm}$ for the outer and inner races, respectively.

Testing was conducted at a constant input shaft speed of about $6 \mathrm{~Hz}$, with torque loads of 30, 50 and $70 \mathrm{Nm}$. Separate testing was performed with the inner and outer race faults. The six test cases are shown in Table 1 . The sampling frequency was set at $131,072 \mathrm{~Hz}$, the switching frequency of the VFD was set at $14 \mathrm{kHz}$, and the control (pseudo line) frequency of the VFD was $24 \mathrm{~Hz}$ (to provide a nominal $6 \mathrm{~Hz}$ input shaft speed for the 8-pole induction motor).

Table 1 Test conditions of the gearbox rig

\begin{tabular}{cccc}
\hline Case & Input shaft speed $(\mathrm{Hz})$ & Load $(\mathrm{Nm})$ & Bearing fault location \\
\hline 1 & 5.78 & 70 & Outer race \\
2 & 5.84 & 50 & Outer race \\
3 & 5.89 & 30 & Outer race \\
4 & 5.78 & 70 & Inner race \\
5 & 5.84 & 50 & Inner race \\
6 & 5.89 & 30 & Inner race \\
\hline
\end{tabular}

\subsubsection{Bearing fault frequencies}

As explained in [1, 32], for a rolling element bearing with a localised defect on one of the races, a series of impacts are generated when each rolling element strikes the defect. Unlike a conventional bearing configuration with a fixed outer race, for a planetary bearing, the outer race rotates at the speed of the planet gear while the inner race rotates in the opposite direction at the speed of the planet carrier, but with a fixed orientation with respect to the load. The kinematic equations for the bearing characteristic defect frequencies in this case are:

Ball pass frequency, outer race $(B P F O)$ :

$$
B P F O=\frac{n}{2}\left[\left(f_{i}-f_{o}\right)\left(1-\frac{d}{D} \cos \phi\right)\right]
$$

Ball pass frequency, inner race $(B P F I)$ :

$$
B P F I=\frac{n}{2}\left[\left(f_{i}-f_{o}\right)\left(1+\frac{d}{D} \cos \phi\right)\right]
$$

Fundamental train frequency (relative to carrier), i.e. cage speed (FTF):

$$
F T F=\frac{1}{2}\left[\left(f_{i}-f_{o}\right)\left(1+\frac{d}{D} \cos \phi\right)\right]
$$

Ball (roller) spin frequency $(B S F)$ : 


$$
B S F=\frac{D\left(f_{i}-f_{o}\right)}{2 d}\left[1-\left(\frac{d}{D} \cos \phi\right)^{2}\right]
$$

where $n$ is the number of rolling elements, $\phi$ is the angle of the load from the radial plane ( $\phi=0$ here), $d$ is the diameter of the roller, and $D$ is the pitch diameter of the bearing. $f_{o}$ and $f_{i}$ are the planet bearing outer and inner race speeds (planet gear and carrier speeds, respectively). In this case, the approximate fault frequencies are: $B P F O=55 \mathrm{~Hz}$ and $B P F I=69 \mathrm{~Hz}$. The inner race fault signal is expected to consist of a series of impulse responses fairly uniform in amplitude (not modulated by rotation of the gear), while the impulse responses in the outer race fault signal are expected to be modulated by the relative frequency of the two races, the rate at which the fault passes through the load zone. Note that for cage speed, most important is the velocity relative to the carrier, which is the rate at which a given rolling element passes through the load zone.

\subsection{3. $\quad$ Measured signals}

For the bearing outer race fault with torque loads of 70, 50 and $30 \mathrm{Nm}$, the raw time signals are plotted in Fig. 10, and their power spectral densities are shown in Fig. 11. From the power spectra, it is clear that these three signals are contaminated by intense EMI generated by the VFD. The spectra contain discrete components at harmonics of the VFD switching frequency (14 kHz), with families of sidebands around these harmonics spaced at the pseudo line frequency $(24 \mathrm{~Hz})$ and twice mains frequency $(100 \mathrm{~Hz})$, as explained in Section 2. The EMI components in fact occupy a very wide frequency range, with these sidebands distributed over several kilohertz. The EMI is also evident in the time records, most notably in the 50 Nm case (Fig. 10(b)), where a number of dropouts can be seen. It was explained in Section 2 that these dropouts arise from large line-to-ground current spikes that occur in alternating directions for each rise and fall of the PWM signal. It was observed here, however, that the dropouts were always in the negative direction ${ }^{3}$, and their magnitudes were quite random, with many small dropouts (some indistinguishable from noise) but relatively few large dropouts.

The signals obtained for the inner race fault cases were very similar to those of the outer race faults, and for brevity have not been shown.

\footnotetext{
${ }^{3}$ It is not known why this is so; it is noted that the EMI shown in acoustic emission signals in [21] appears to feature dropouts in both directions.
} 
a

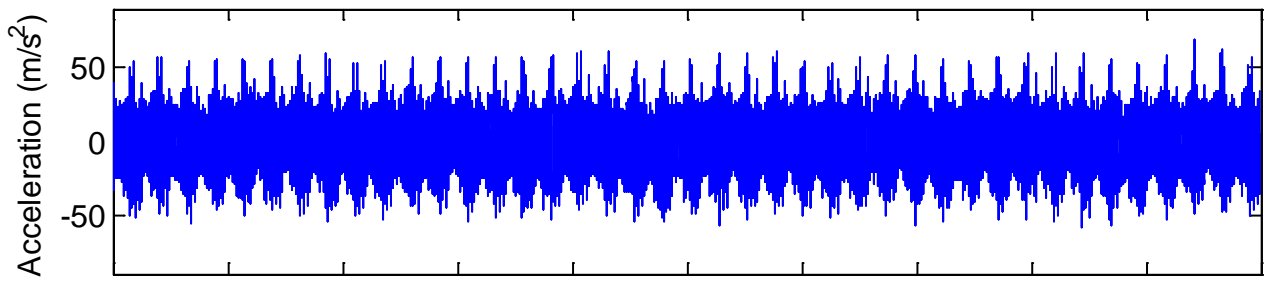

b

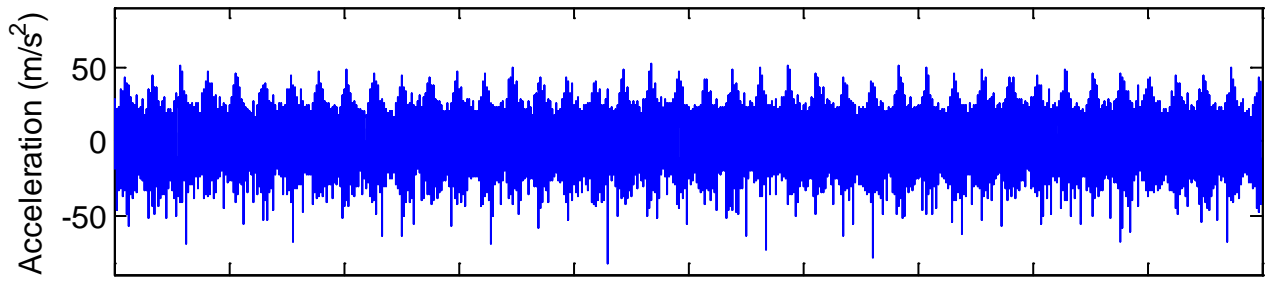

C

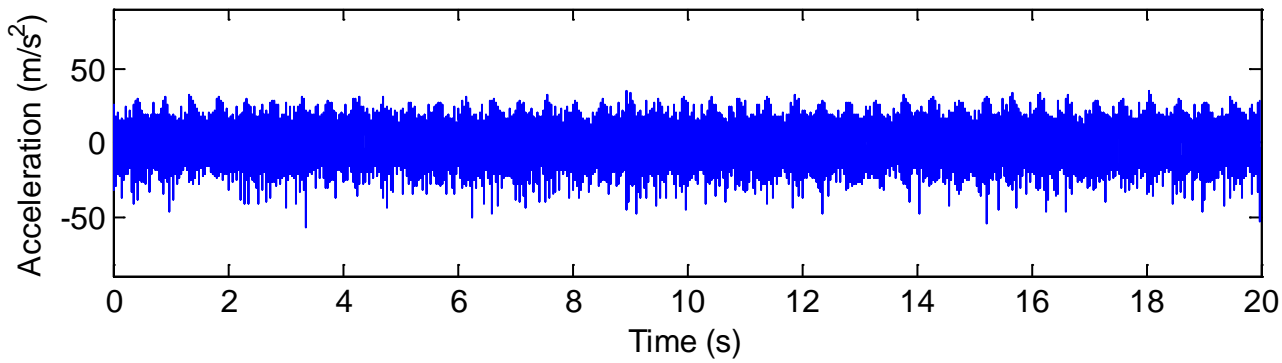

Fig. 10 Time waveforms of ORF signals at torque loads of (a) $70 \mathrm{Nm}$, (b) $50 \mathrm{Nm}$, and (c) $30 \mathrm{Nm}$

a

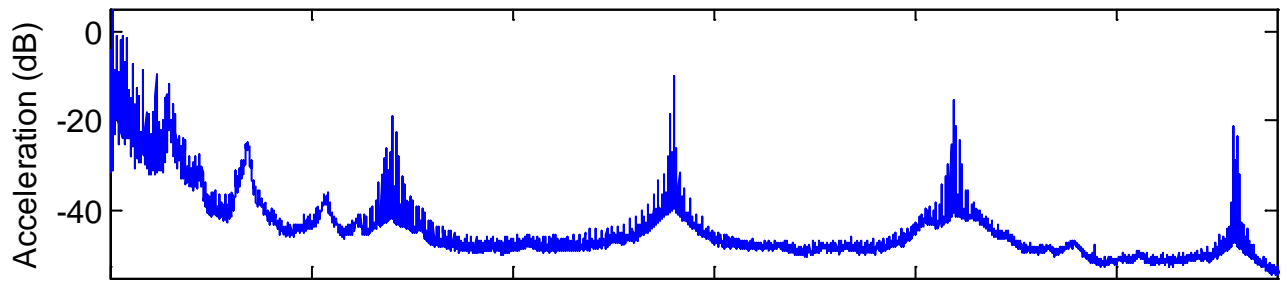

b

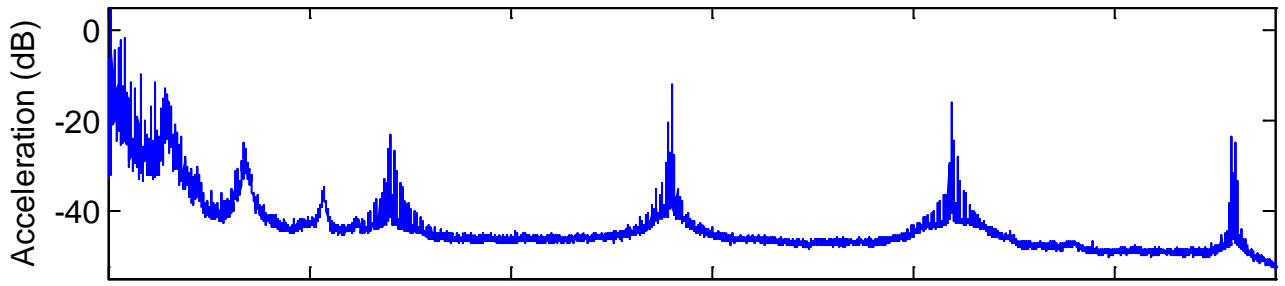

C

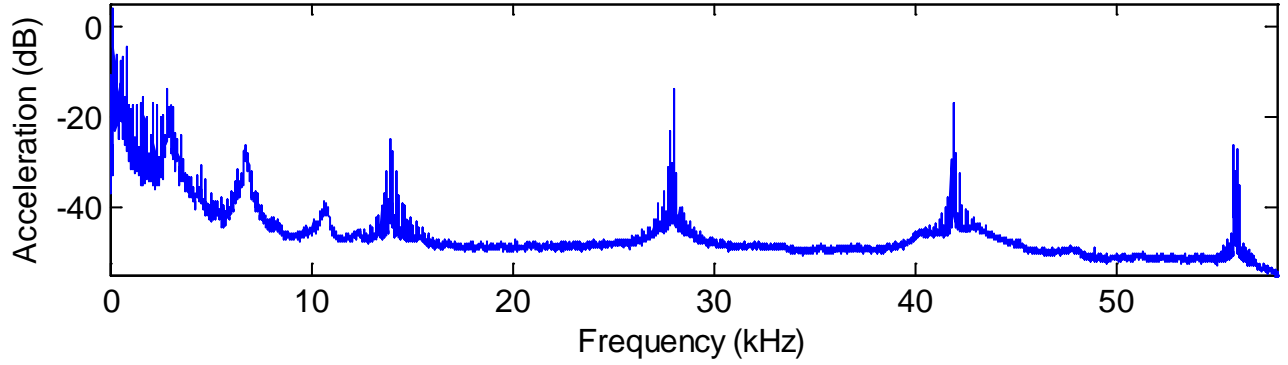

Fig. 11 PSDs of ORF signals at torque loads of (a) $70 \mathrm{Nm}$, (b) $50 \mathrm{Nm}$, and (c) $30 \mathrm{Nm}$

\subsection{Simulated data}

To further investigate the EMI phenomenon and its effect on bearing diagnostics, a number of simulated digital signals were produced in Matlab ${ }^{\circledR}$. The signals were designed to qualitatively 
replicate the experimental data outlined in Section 4.1. Each signal comprised an additive combination of the following components:

1. Bearing fault signal, represented by a series of uniform impulse responses with a slight random variation in spacing.

2. Electromagnetic interference from a pulse-width modulated signal, represented by a series of delta functions to replicate the dropout effect discussed in Section 2.2.

3. Low frequency harmonics to represent harmonics of the input shaft, planet carrier and planetary gearmesh frequency from the test rig outlined in Section 4.1.1.

4. White Gaussian noise to represent the relatively flat noise level observed in the measured vibration signals (shown in Section 4.1.3).

\subsubsection{Bearing fault signal}

The bearing fault signal was represented by a series of uniform single degree-of-freedom impulse responses, each described (in displacement terms) by:

$$
x(t)=e^{(-t / \tau)} \sin \left(2 \pi f_{n} t\right)
$$

where $f_{n}$ is the frequency of the resonance and $\tau$ is the damping time constant. The generated signal obtained using Eq. (7) and then double differentiating to give acceleration - was ultimately sampled at $131,072 \mathrm{~Hz}$, but was first generated at five times that frequency (then decimated) to avoid aliasing. The natural frequency was set to $14 \mathrm{kHz}$, and the damping time constant, at $0.197 \mathrm{~ms}$, was chosen to give a $3 \mathrm{~dB}$ bandwidth for the resonance of $1 / 6^{\text {th }}$ octave, or $1,616 \mathrm{~Hz}$. The complete signal was created by repeating the generated impulse response, with the average spacing between successive pulses set to give a fault frequency of $69 \mathrm{~Hz}$. To represent random slip, this spacing was adjusted using a uniformly distributed random number over the range $\pm 1 \%$ of the average.

\subsubsection{Electromagnetic interference from a pulse-width modulated signal}

As with the bearing fault signal, the EMI signal was generated at five times the ultimate sample rate (131,072 Hz), then decimated. Trailing-edge modulation, as depicted in Fig. 1(b), was used, with a switching frequency of $8 \mathrm{kHz}$ (to represent a midpoint in the typical $2-15 \mathrm{kHz}$ range [13]) and a pseudo line frequency of $24 \mathrm{~Hz}$. To approximate the line-to-ground current transients discussed in Section 2.2, delta functions were used since the frequency content of the transients was considered to be above the frequency range of interest. This equates to taking the derivative of the PWM signal, or a multiplication by $j \omega$ in the frequency domain, which explains why the spectral characteristics observed in the measured signals match the general description of PWM signals outlined in Section 2.1. Using the derivative is also very computationally efficient. To match the characteristics of the dropouts in the measured signals discussed in Section 4.1.3, only the negative delta functions were used. This process is shown conceptually in Fig. 12.

It was found that the magnitude of the dropouts in the measured signals was quite random, with many small dropouts but relatively few large dropouts. This was incorporated into the simulation by replacing each (negative) delta function with a random number of exponential distribution. A low-pass filter was then applied to the signal to simulate a typical anti-aliasing filter, resulting in a 
very similar effect to the sawtooth filter response observed in the measured signals. This is illustrated in Fig. 13, which shows the complete simulated EMI signal and a zoomed version showing this filter response.

a

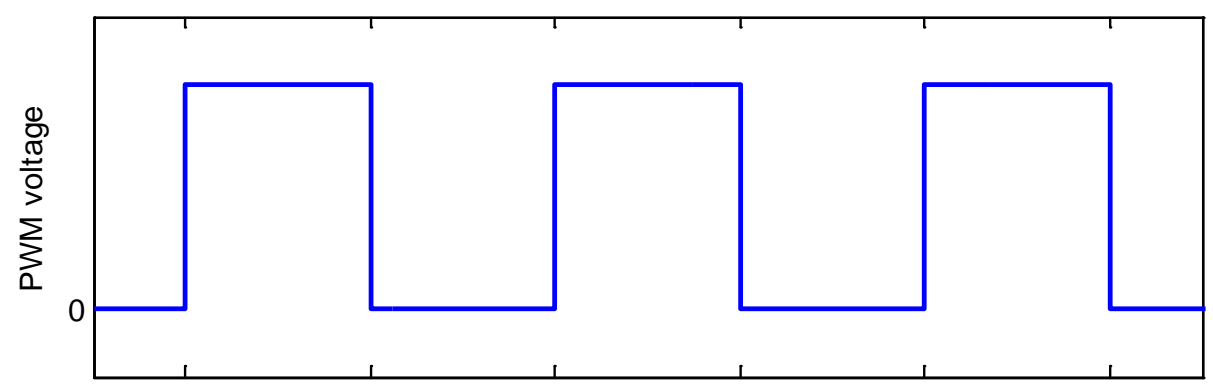

b

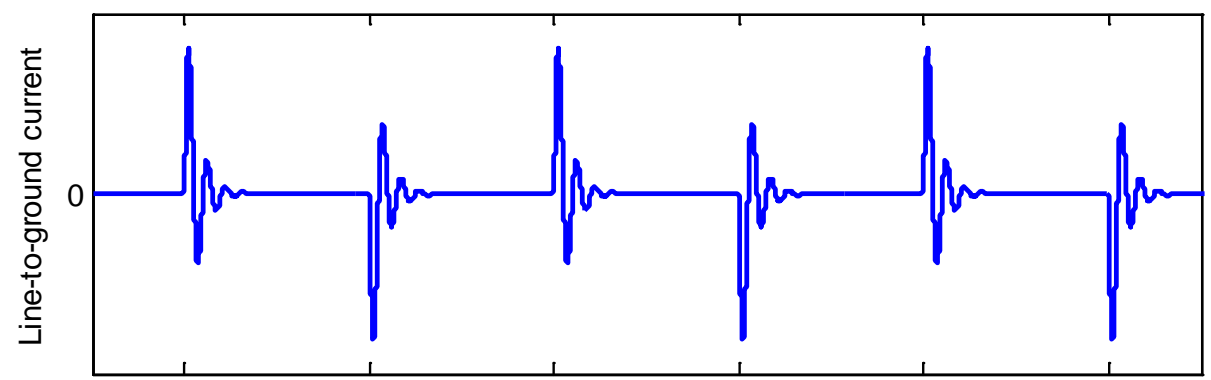

C

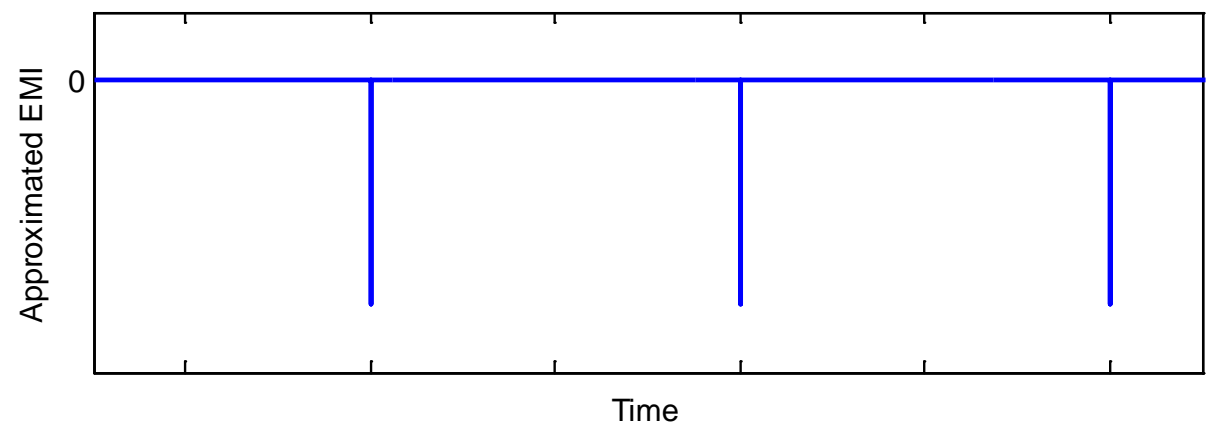

Fig. 12 Simulation of EMI dropouts; (a) PWM voltage; (b) line-to-ground current transients; (c) delta functions approximating EMI (only negative current spikes in this case) 
a

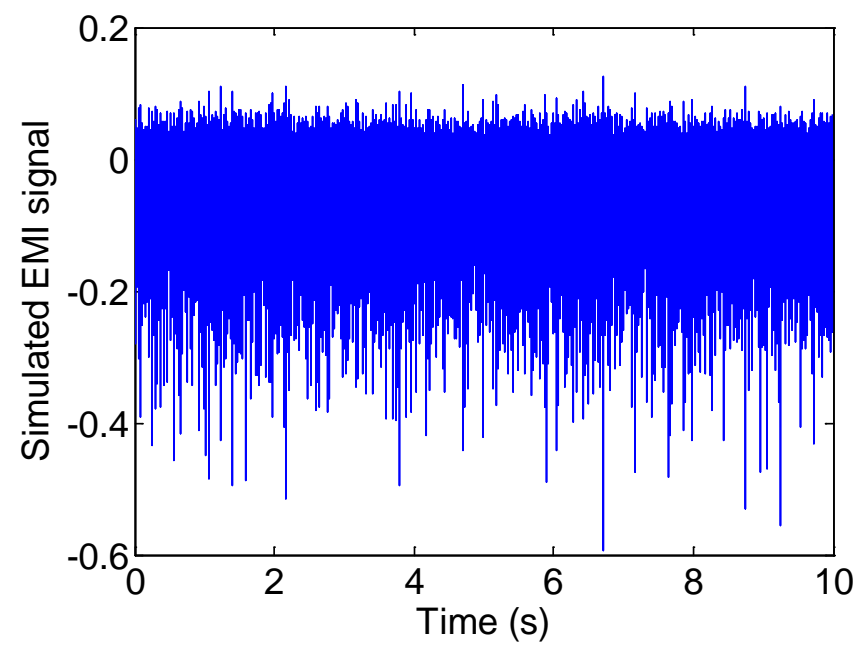

b

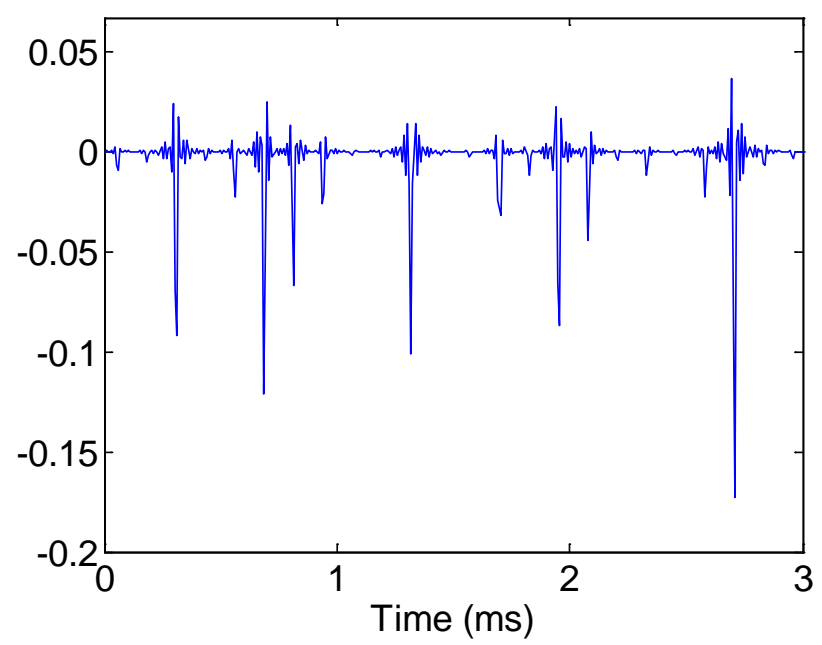

Fig. 13 Simulated EMI signal after first scaling with exponentially-distributed random number sequence, then low-pass filtering; (a) complete signal; (b) zoomed signal showing sawtooth filter effect on dropouts

\subsubsection{Low frequency harmonics}

To provide a rich combination of discrete components over the first few kilohertz of the generated signal spectrum, the first 30 harmonics of the input shaft, planet carrier and planetary gearmesh frequency were included in the signal as sinusoidal components with random phase. The amplitude of the harmonics was set to decay with increasing harmonic number, $n$, at a rate of $1 / n^{3 / 2}$. The kinematic relationships of the test rig in Section 4.1.1 were used, with an input shaft speed of $5.8 \mathrm{~Hz}$ specified, giving planet carrier and gearmesh frequencies of approximately $2.1 \mathrm{~Hz}$ and $165 \mathrm{~Hz}$, respectively.

\subsubsection{White Gaussian noise}

In measured vibration signals, 'noise' is rarely white but is rather shaped by the transfer function from the source to the measurement point. Yet white noise was added to the generated signal in this case to represent the relatively flat noise level observed in the measured vibration signals shown in Section 4.1.3.

\subsubsection{Complete simulated signal}

To obtain the complete simulated signal, these four components were added together with a weighting apportioned to each component. A number of different signals were generated in which all the weightings were held constant except that of the bearing fault signal, which was varied to study the performance of bearing diagnostic algorithms in the presence of EMI and with various signal-to-noise ratios (SNRs). The SNR definition used here is the ratio of power of the bearing fault signal to that of the remainder of the signal, determined over a band of $512 \mathrm{~Hz}$ centred on $f_{n}(14 \mathrm{kHz})$. The generated signal had a 10-second duration. An example of a complete signal is shown in Fig. 14, where the resonance at $14 \mathrm{kHz}$ can be clearly seen in the spectrum. Note that this represents an exaggerated power ratio of approximately $2.7(4.3 \mathrm{~dB})$, which is much larger than that required to achieve a successful diagnosis (using the Fast Kurtogram, for example). Also clearly seen in the figure are the very strong EMI components at harmonics of the switching frequency $(8 \mathrm{kHz})$, each with a tight 
grouping of sidebands spaced at $24 \mathrm{~Hz}$, and a widely distributed group that is spaced at $48 \mathrm{~Hz}$ and runs into the noise floor. The dropouts from this EMI can also be seen in the time signal, which shows a skewed distribution and a few larger dropouts protruding from the rest.

For the results presented in Section 5, the weighting of the fault-related signal component was set to give an SNR of approximately $0.46(-3.4 \mathrm{~dB})$. The obtained SNR threshold values indicating when each method was found to be successful are given in the discussion in Section 6.1.2.

a

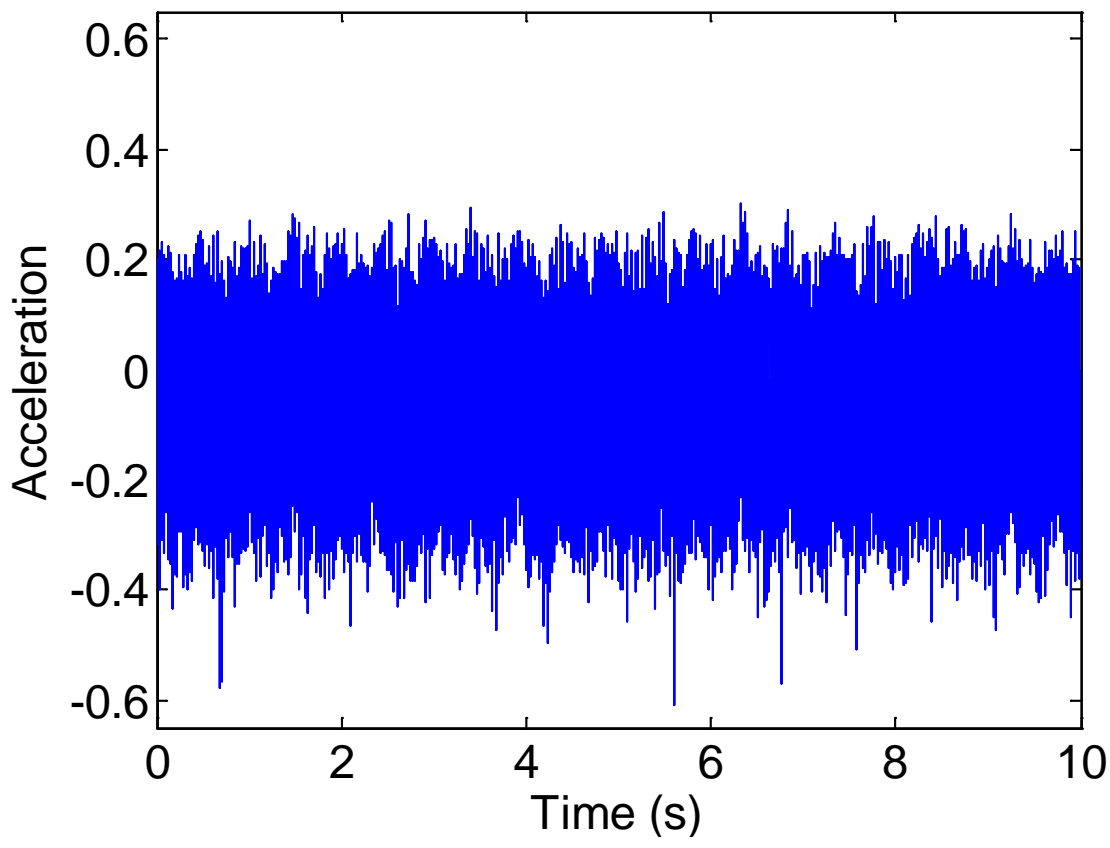

$\mathrm{b}$

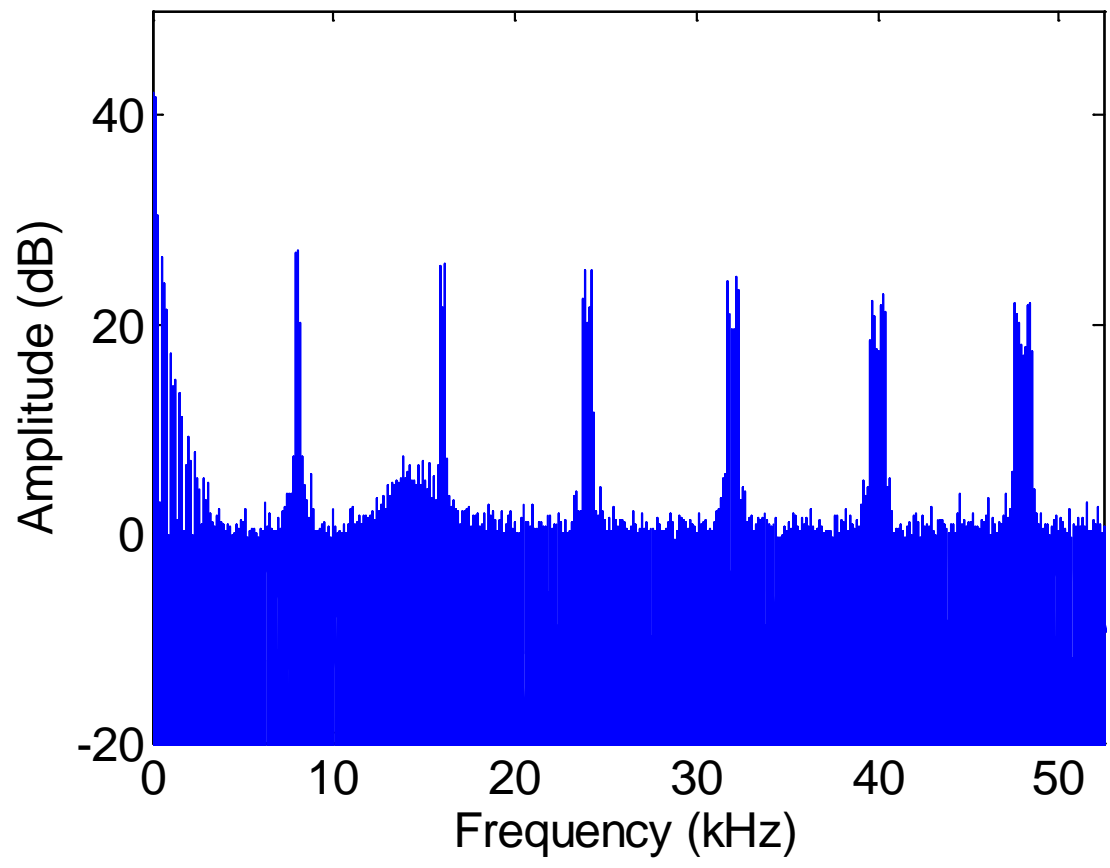

Fig. 14 Simulated bearing fault signal with strong EMI; (a) time plot; (b) amplitude spectrum

\section{Results}

In this section, the measured and simulated bearing fault signals described in Section 4 are 
processed by the three demodulation band selection methods outlined in Section 3, and the performance of the techniques is discussed. The techniques are applied to the raw signals, with no preprocessing, as mentioned in Section 2.4. The Fast Kurtogram was applied down to a bandwidth of $512 \mathrm{~Hz}$ (Level 7), and the Protrugram and Optimised SK were applied with a step size of $100 \mathrm{~Hz}$ and bandwidth of $512 \mathrm{~Hz}$ (which, at about $7 \times \mathrm{BPFI}$, is broader than strictly required). Results from the measured $30 \mathrm{Nm}$ load cases (3 and 6 in Table 1) have been omitted for brevity, but the diagnostic outcomes for all tests are summarised in Section 6.1.

\subsection{Experimental data results}

\subsection{1. $\quad$ Outer race fault (ORF) detection}

The three methods were first applied to the bearing fault signal for Case 1 (70 Nm torque, outer race fault). The Fast Kurtogram results are shown in Fig. 15, where the determined demodulation band parameters are: centre frequency $(C F)$ of $64 \mathrm{kHz}$, and bandwidth $(B W)$ of 1,024 $\mathrm{Hz}$. From the corresponding envelope spectrum, the fault information can be clearly identified. It is perhaps worth noting here that this frequency band, very close to the Nyquist frequency $(65,536 \mathrm{~Hz})$, would certainly contain aliased components. But as observed in [12], aliasing should not adversely affect envelope analysis, since the repetition frequency of the impulses is sought, and not their individual frequency content.

A plot of the Protrugram for Case 1 is given in Fig. 16(a), giving an optimal CF of 14,456 Hz, which is near the VFD switching frequency of $14 \mathrm{kHz}$. As shown in Fig. 16(b), the related envelope spectrum fails to diagnose the bearing fault, and is dominated instead by EMI-related components of 24 and $100 \mathrm{~Hz}$, and their harmonics.

It is interesting to note that the region of the Kurtogram corresponding to the band selected by the Protrugram $(14,200-14,712 \mathrm{~Hz}$ ) is one of relatively low kurtosis (dark blue on the colour scale). Likewise, the band selected by the Kurtogram $(63,488-64,512 \mathrm{~Hz})$ shows a very low kurtosis in the Protrugram. It appears that the problem for the Protrugram in this case is that the EMI signal is very well structured, with a periodic modulation pattern giving strong, discrete components (and thus high kurtosis) in the envelope spectrum. This is clearly reflected in the Protrugram itself, which shows spikes in kurtosis at the VFD switching frequency harmonics. Barszcz and Jabłoński stated in [9] that maxima with steep edges in the Protrugram should be ignored because they are caused by harmonic components rather than the typical signature of a faulty bearing, which should be indicated by maxima with 'gentle slopes'. However, this selection criterion is quite ambiguous, and certainly difficult to apply in this instance. As shown in Fig. 16, the Protrugram has a number of 'hill-like' shapes distributed over a broad frequency range, all of which could be treated as candidates for demodulation and further analysis. For this reason, the authors here select the centre frequency with the maximum kurtosis value in the Protrugram, rather than checking every hill-like form.

The Optimised SK results for Case 1 are shown in Fig. 17, giving a CF of 64,656 Hz, which is very close to the Fast Kurtogram results $(C F=64 \mathrm{kHz}$ and $B W=1,024 \mathrm{~Hz})$. Not surprisingly, the corresponding envelope spectrum is very similar to that obtained using the Fast Kurtogram (Fig. 
15(b)), showing clear BPFO harmonics.

a

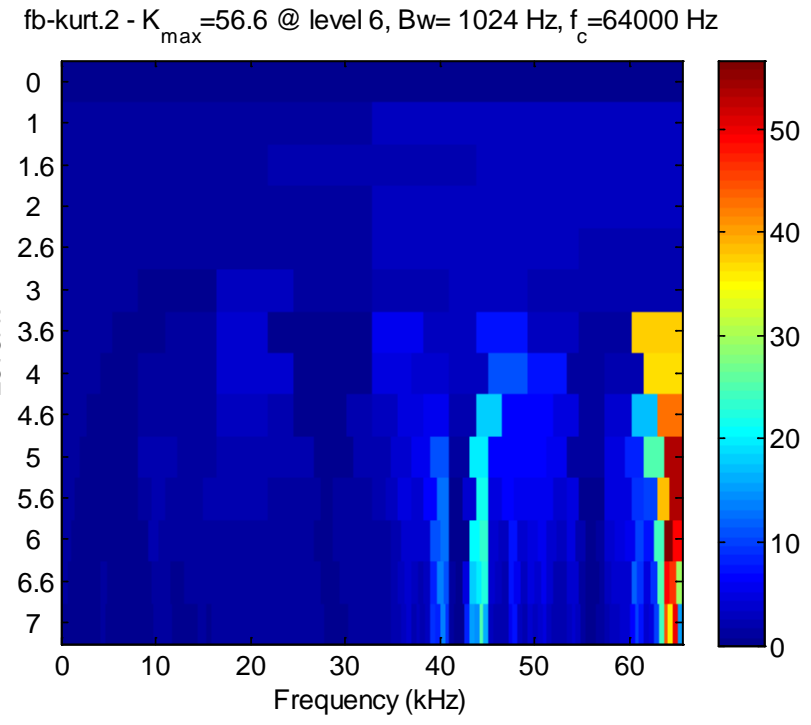

$\mathrm{b}$

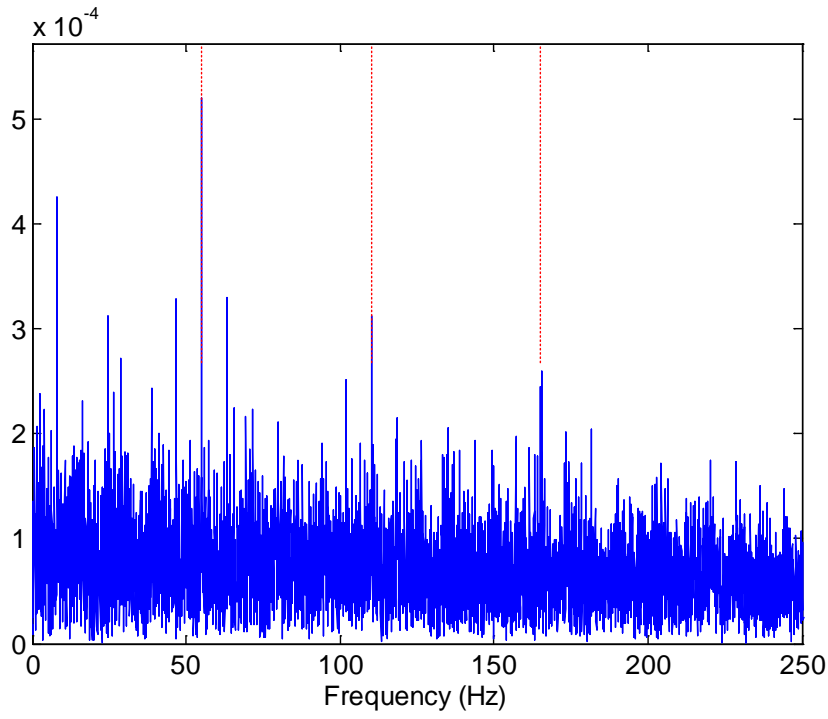

Fig. 15 Fast Kurtogram results for Case 1 (70 Nm, ORF); (a) Fast Kurtogram; (b) squared envelope spectrum (cursors at BPFO harmonics)

a

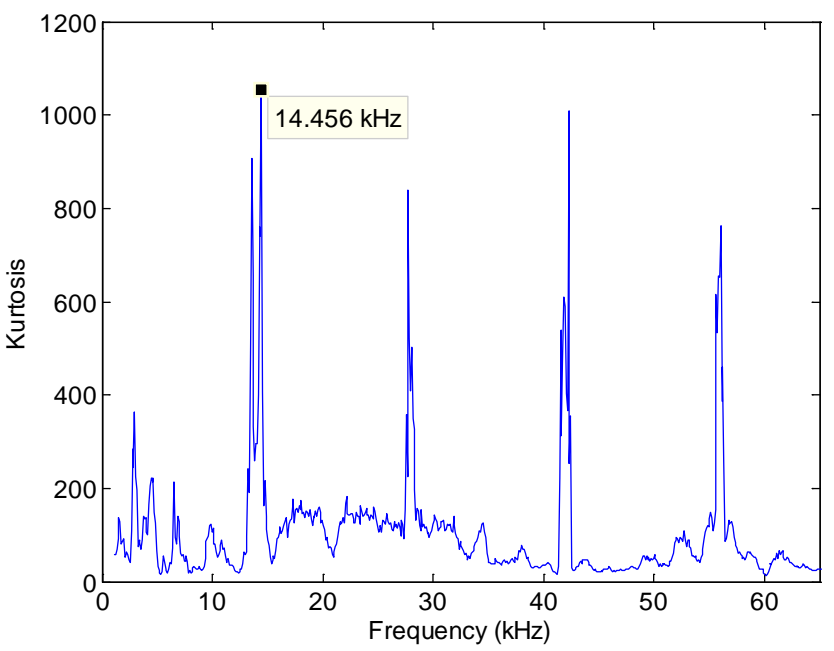

$\mathrm{b}$

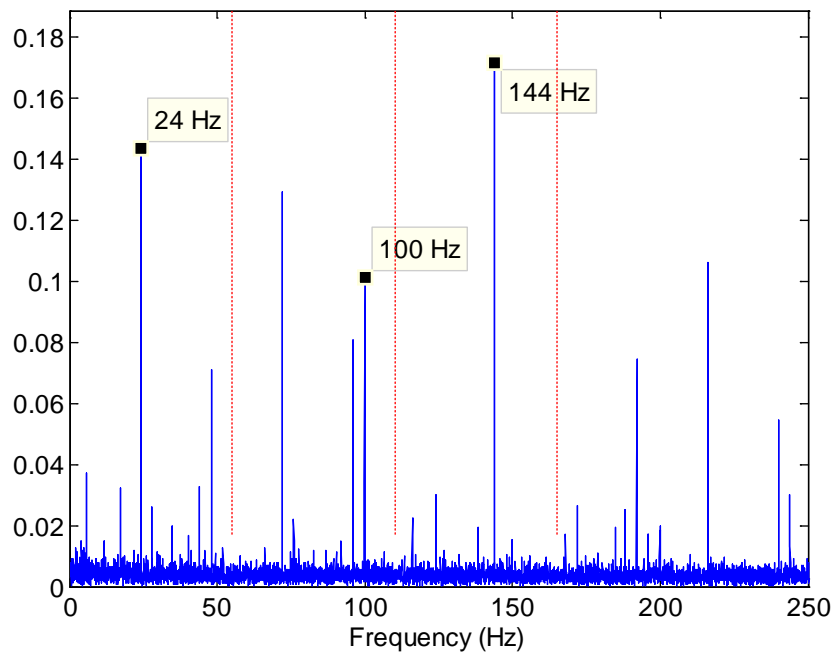

Fig. 16 Protrugram results for Case 1 (70 Nm, ORF); (a) Protrugram; (b) squared envelope spectrum (cursors at BPFO harmonics) 

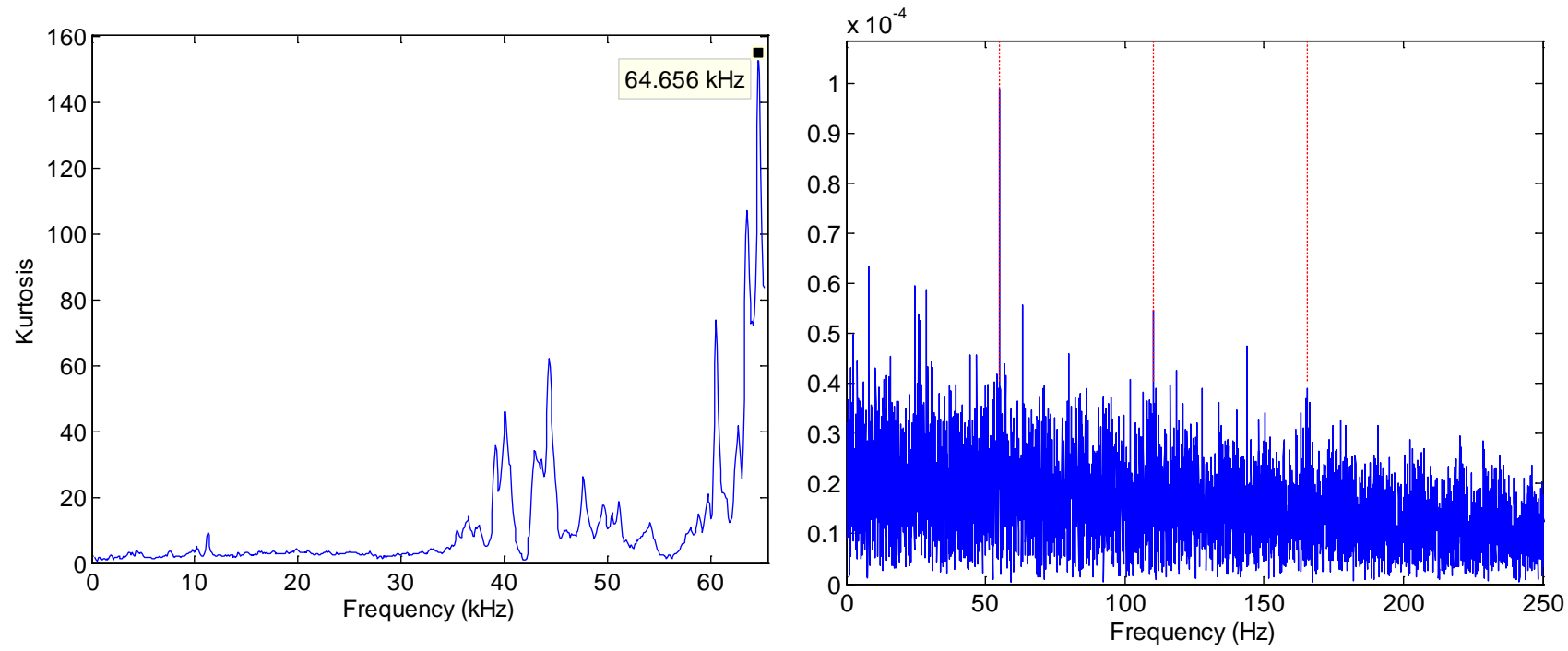

Fig. 17 Optimised SK results for Case 1 (70 Nm, ORF); (a) SK; (b) squared envelope spectrum (cursors at BPFO harmonics)

Shown in Fig. 18(a) is the Fast Kurtogram for Case 2 (50 Nm torque, outer race fault), giving $C F=19,115 \mathrm{~Hz}$ and $B W=5,461 \mathrm{~Hz}$. However, as shown in Fig. 18(b), the squared envelope spectrum is dominated by the EMI component at $100 \mathrm{~Hz}$ (twice mains frequency of $50 \mathrm{~Hz}$ ) and its second harmonic, and thus fails to reveal the bearing fault. Components at harmonics of the input shaft speed, about $6 \mathrm{~Hz}$, can also be seen throughout the spectrum.

The plot of the Protrugram for Case 2 is presented in Fig. 19(a), showing that an optimal CF of 26,256 Hz is selected. The corresponding envelope spectrum in Fig. 19(b) fails to recognise the fault, and is dominated by EMI-related components of $100 \mathrm{~Hz}$ and their harmonics.

Fig. 20(a) shows the Optimised SK for Case 2, giving a $C F$ of 40,356 $\mathrm{Hz}$, in contrast to the Kurtogram values of $C F=19,115 \mathrm{~Hz}$ and $B W=5,461 \mathrm{~Hz}$. The SK plot in Fig. 20(a), with a very sharp spike, highlights the value in being able to fine-tune the centre frequency selection by using small step sizes. The envelope spectrum, in Fig. 20(b), shows clear harmonics of BPFO, albeit with a strong $100 \mathrm{~Hz}$ EMI component. By comparison, the envelope spectrum obtained using the Fast Kurtogram (Fig. 18(b)) was completely unsuccessful in diagnosing the fault. It is interesting to note that the Fast Kurtogram of Fig. 18(a) would have delivered a band just over $40 \mathrm{kHz}$ if it had been restricted to a Level 7 bandwidth $(B W=512 \mathrm{~Hz})$ - i.e. 'looking along one row' - and this probably would have given a successful diagnosis. The Kurtogram's problem in this application is in not restricting the bandwidth and thus allowing too much interference to pass through. 
$\mathrm{a}$

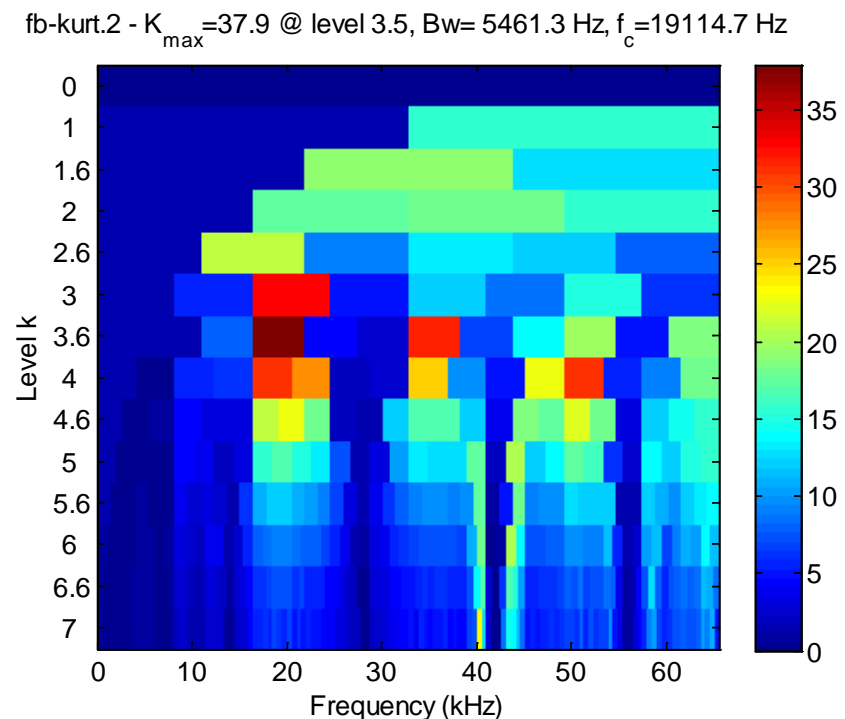

$\mathrm{b}$

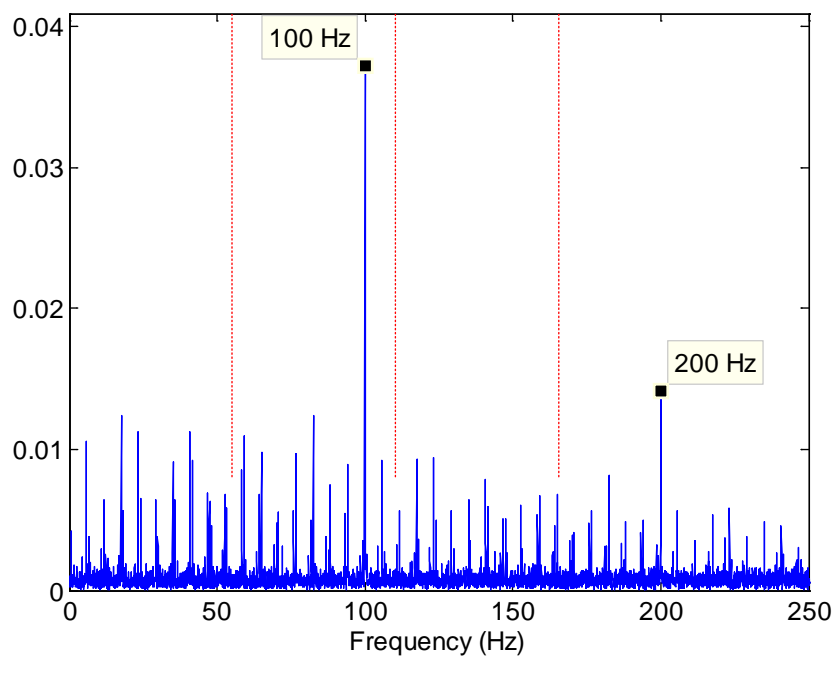

Fig. 18 Fast Kurtogram results for Case 2 (50 Nm, ORF); (a) Fast Kurtogram; (b) squared envelope spectrum (cursors at BPFO harmonics)

a

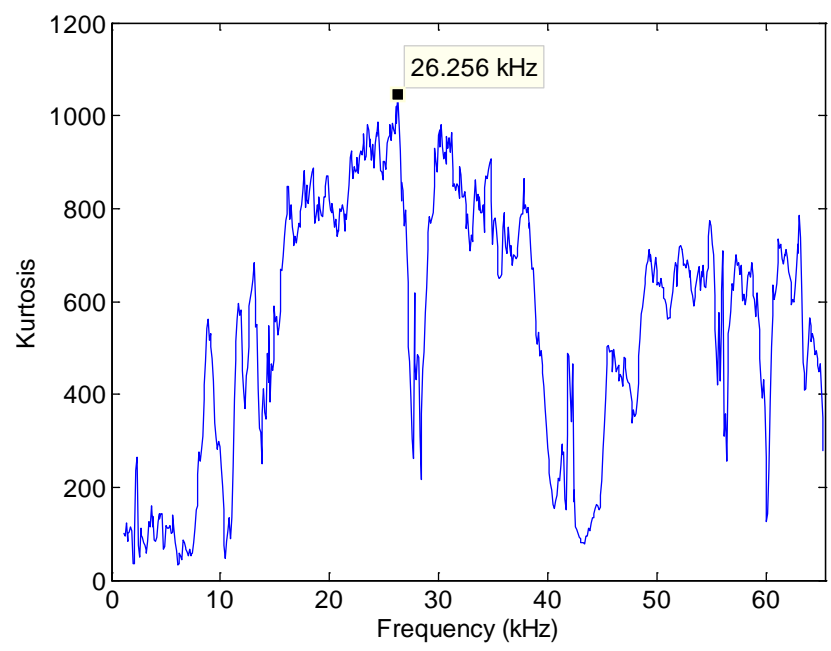

$\mathrm{b}$

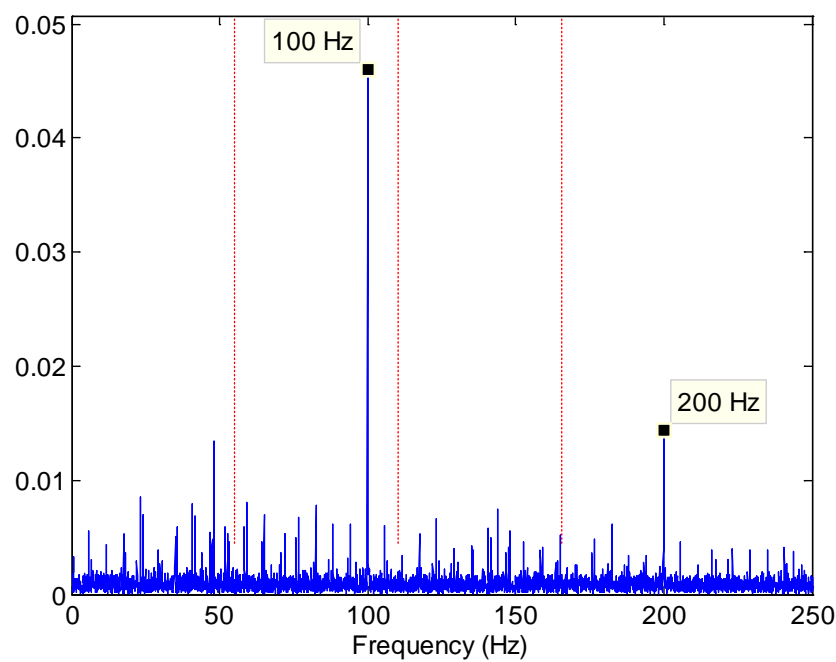

Fig. 19 Protrugram results for Case 2 (50 Nm, ORF); (a) Protrugram; (b) squared envelope spectrum (cursors at BPFO harmonics) 

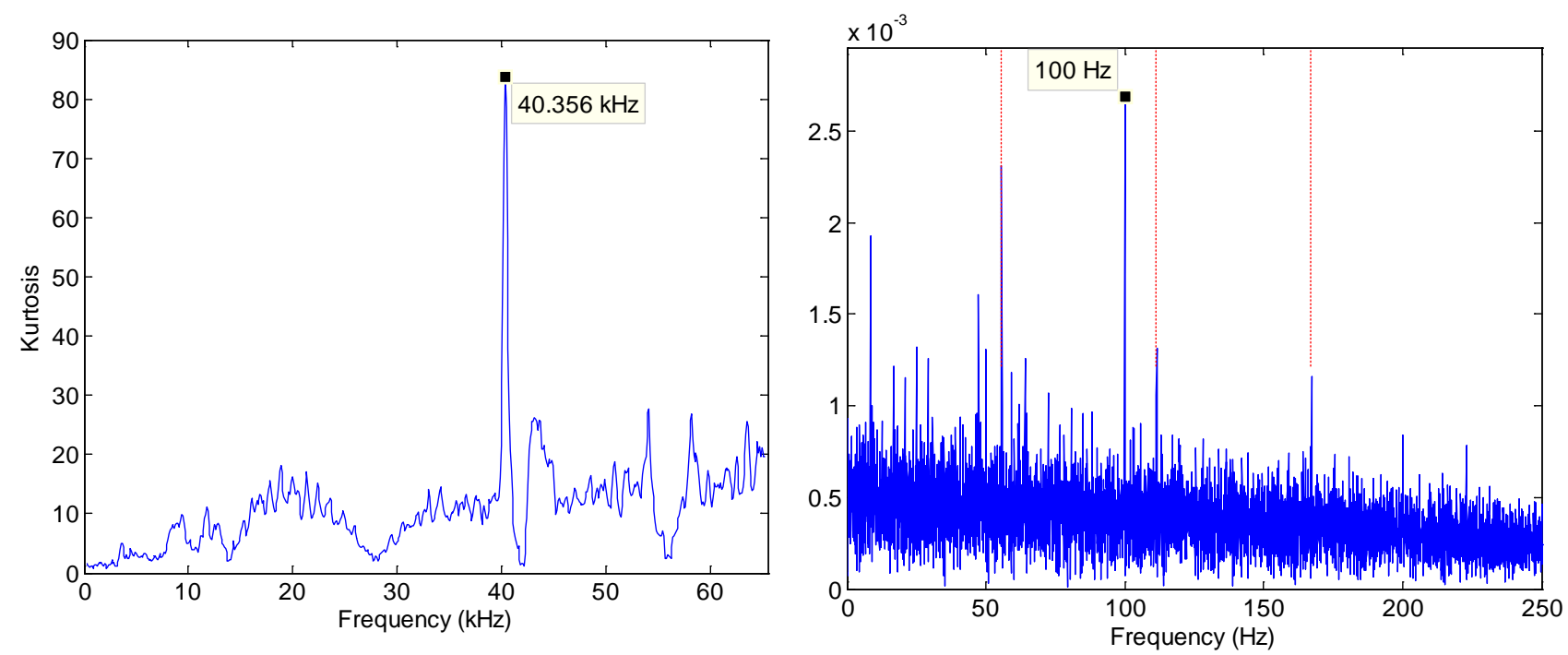

Fig. 20 Optimised SK results for Case 2 (50 Nm, ORF); (a) SK; (b) squared envelope spectrum (cursors at BPFO harmonics)

\subsubsection{Inner race fault (IRF) detection}

Shown in Fig. 21(a) is the Fast Kurtogram for Case 4 (70 Nm torque, inner race fault), giving demodulation band parameters of $C F=64,512 \mathrm{~Hz}$ and $B W=2,048 \mathrm{~Hz}$, similar to Case 1 with the outer race fault. The corresponding envelope spectrum in Fig. 21(b) gives a clear inner race fault diagnosis. The BPFI harmonics in the spectrum are modulated by the planet carrier speed $(2.05 \mathrm{~Hz})$, and by twelve times the carrier speed, the reason for which is not immediately clear.

The Protrugram results are shown in Fig. 22. The optimal CF, at $42,256 \mathrm{~Hz}$, is near the third harmonic of the $14 \mathrm{kHz}$ switching frequency. The related envelope spectrum is dominated by the EMI components of $24 \mathrm{~Hz}$ and $100 \mathrm{~Hz}$, and their harmonics, with only weak components at the BPFI harmonics. However, it is noted in Fig. 22(a) that two visible 'humps' are apparent between 38 and $46 \mathrm{kHz}$ in the Protrugram. As mentioned in Section 5.1.1 and in ref. [9], such a structure may indicate the sought bearing fault. Fig. 23 shows the envelope spectrum obtained after demodulating a band around the maximum of the second hump $(C F=43,656 \mathrm{~Hz})$, and here the bearing fault can be easily diagnosed.

The Optimised SK for Case 4 is shown in Fig. 24(a), giving a CF of 63,656 Hz, which, as with Case 1 , is very close to the band selected by the Fast Kurtogram $(C F=64,512 \mathrm{~Hz}$ and $B W=2,048 \mathrm{~Hz})$ in Fig. 21(a). The corresponding envelope spectrum in Fig. 24(b) shows clear BPFI harmonics, surrounded by sidebands at $1 \times$ and $12 \times$ the planet carrier speed, much like that obtained using the Fast Kurtogram. 
$\mathrm{a}$

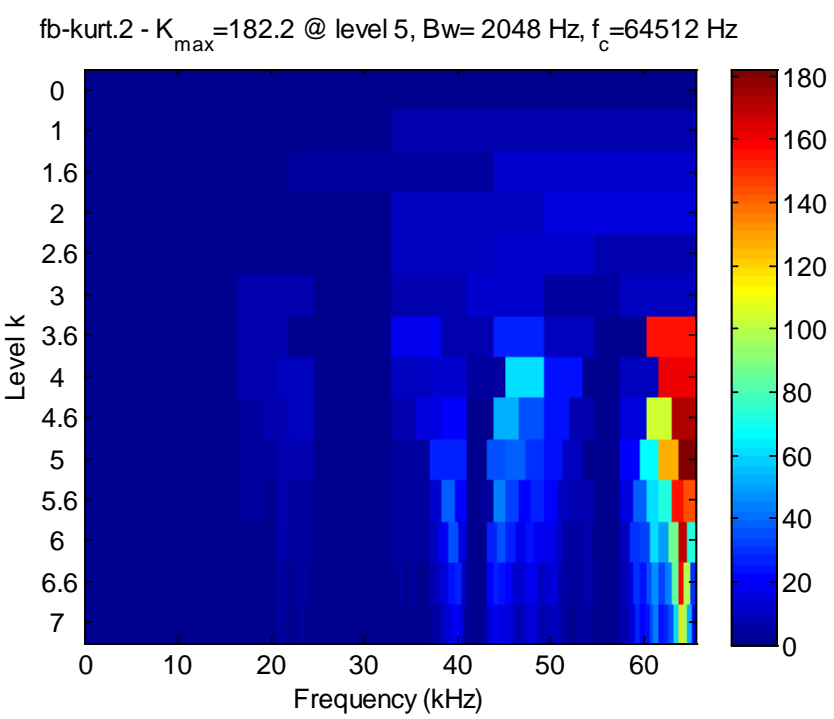

b

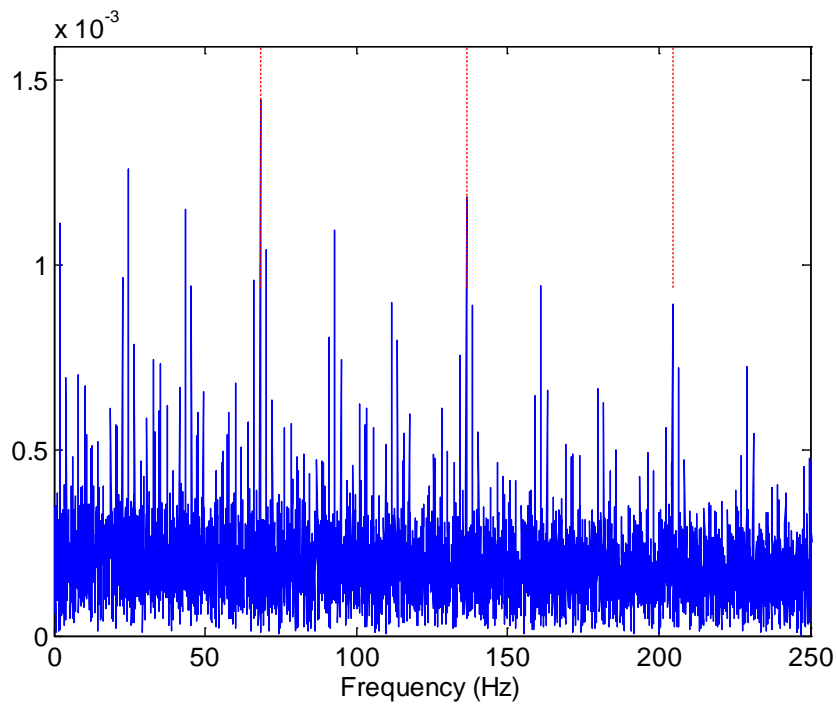

Fig. 21 Fast Kurtogram results for Case 4 (70 Nm, IRF); (a) Fast Kurtogram; (b) squared envelope spectrum (cursors at BPFI harmonics)

$\mathrm{a}$

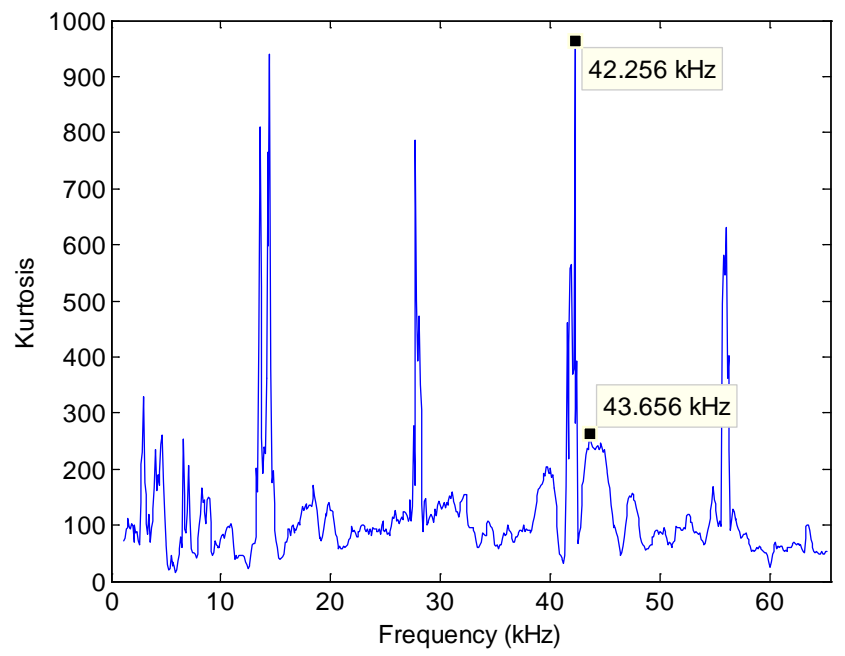

b

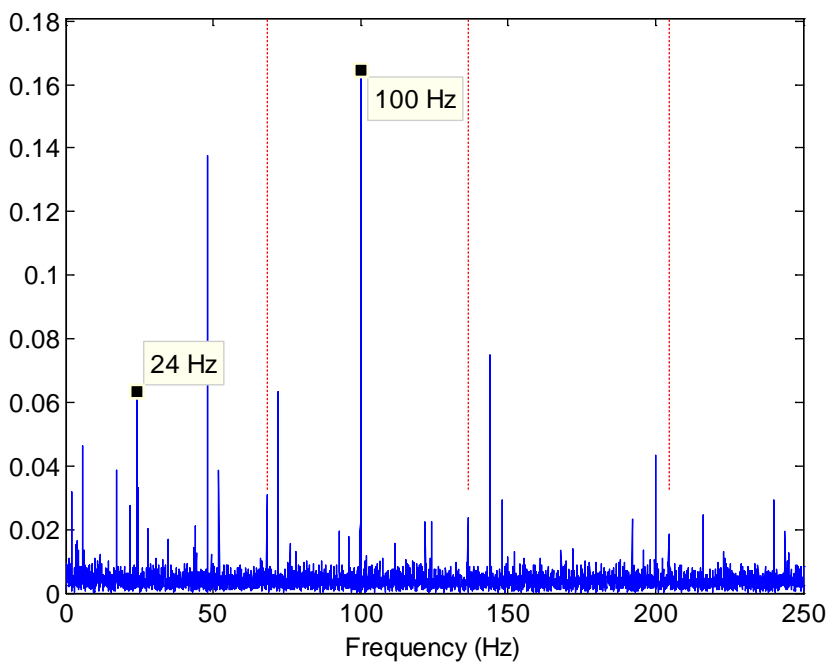

Fig. 22 Protrugram results for Case 4 (70 Nm, IRF); (a) Protrugram; (b) squared envelope spectrum (cursors at BPFI harmonics) 


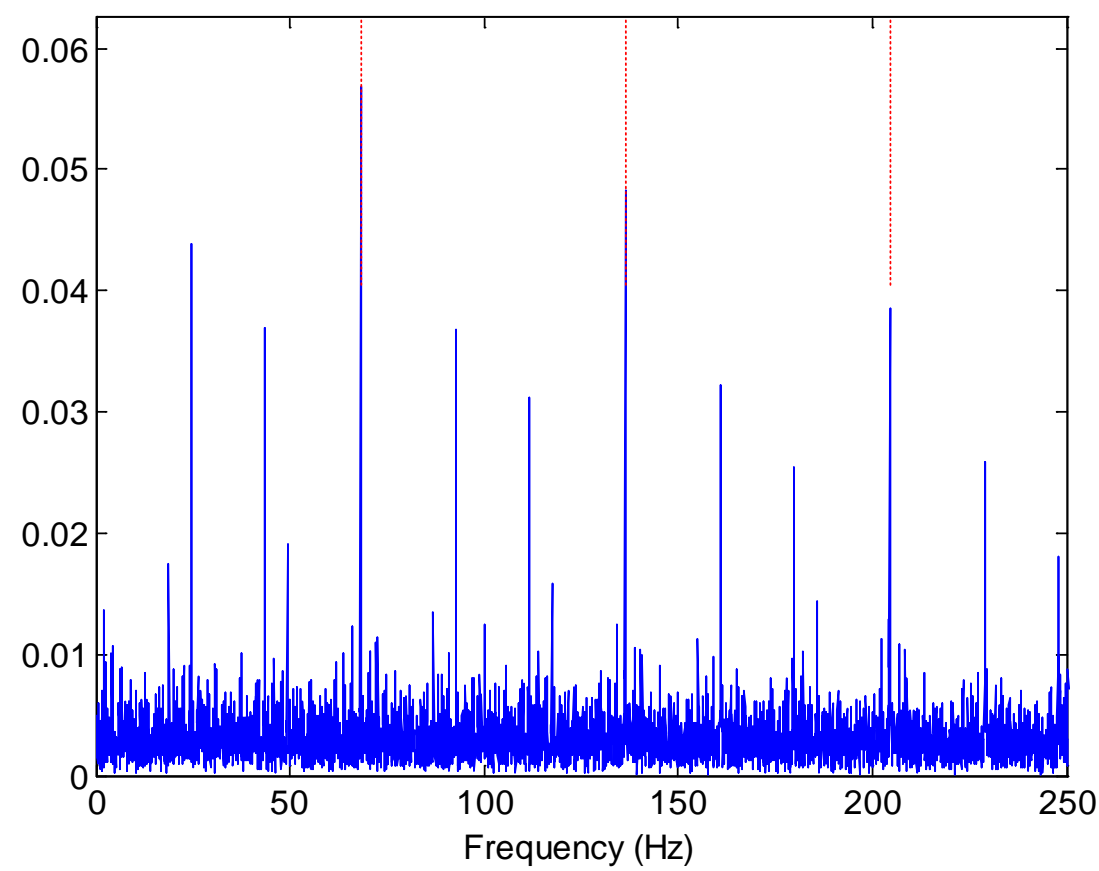

Fig. 23 Squared envelope spectrum from Protrugram for Case 4 (70 Nm, IRF) based on maximum of the 'hump' (cursors at BPFI harmonics)

a

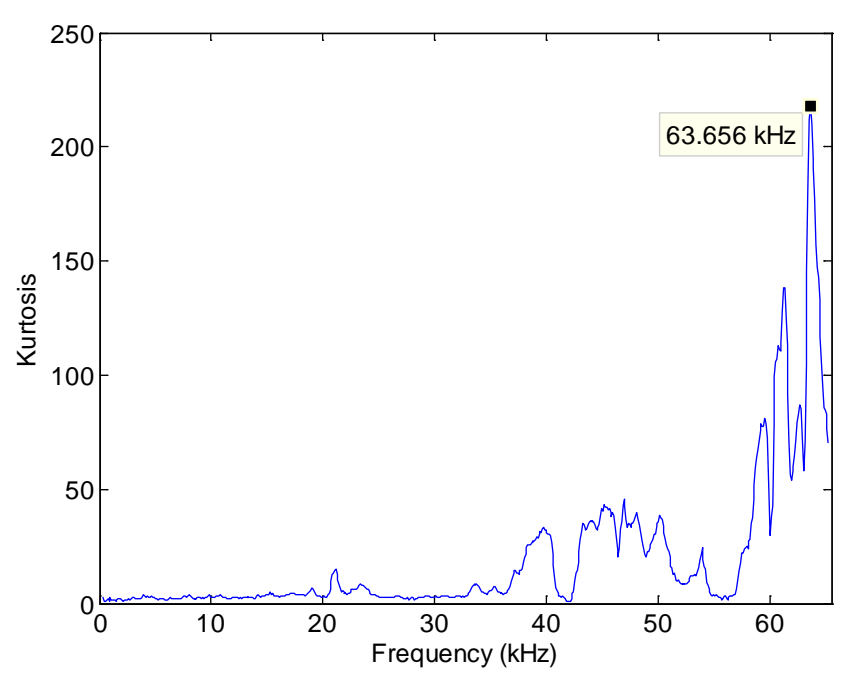

b

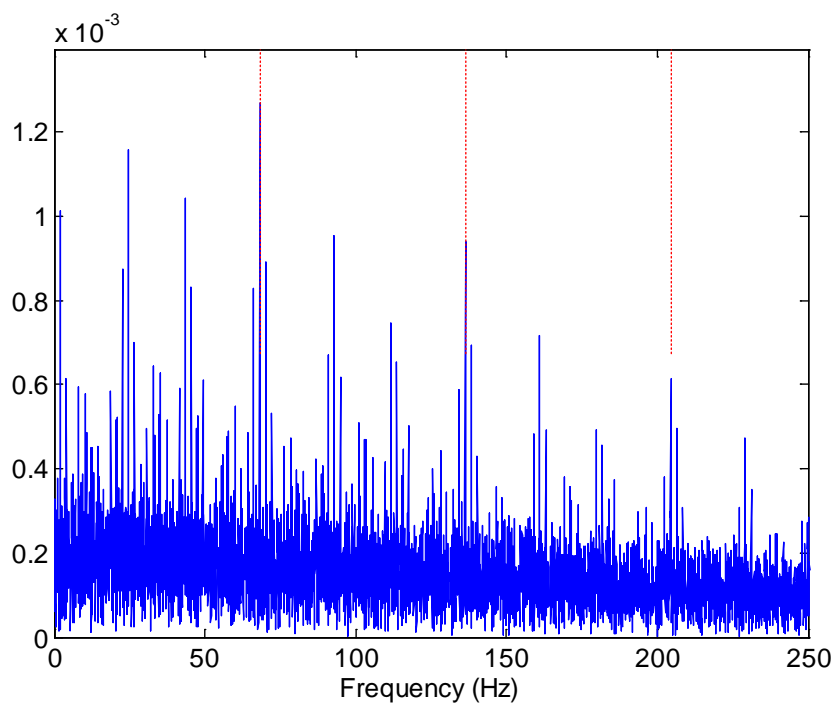

Fig. 24 Optimised SK results for Case 4 (70 Nm, IRF); (a) SK; (b) squared envelope spectrum (cursors at BPFI harmonics)

Fig. 25(a) shows the Fast Kurtogram for Case 5 (50 Nm torque, inner race fault), which gives $C F=47,104 \mathrm{~Hz}$ and $B W=4,096 \mathrm{~Hz}$. The fault information can be extracted from the envelope spectrum in Fig. 25(b), but the EMI component at $100 \mathrm{~Hz}$ is dominant, and the diagnosis is not particularly clear. The Protrugram for this case is shown in Fig. 26(a). The optimal CF is 27,756 Hz, which is near the second harmonic of the switching frequency. The related envelope spectrum, in Fig. 26(b), is dominated by the EMI components of $24 \mathrm{~Hz}, 100 \mathrm{~Hz}$ and their harmonics.

The Optimised SK results for Case 5 are shown in Fig. 27. The determined $C F$ of 47,756 $\mathrm{Hz}$ 
corresponds closely to that obtained with the Fast Kurtogram $(C F=47,104 \mathrm{~Hz} ; B W=4,096 \mathrm{~Hz})$. Interestingly, although EMI components of 24 and $100 \mathrm{~Hz}$ are still dominant in the envelope spectrum of Fig. 27(b), it gives a much clearer diagnosis than that provided by the Fast Kurtogram (Fig. 25(b)). This strengthens the argument that minimising the bandwidth maximises the SNR with this sort of interference.

$\mathrm{a}$

fb-kurt.2 - $\mathrm{K}_{\max }=45.5 @$ level 4, Bw= $4096 \mathrm{~Hz}, \mathrm{f}_{\mathrm{c}}=47104 \mathrm{~Hz}$

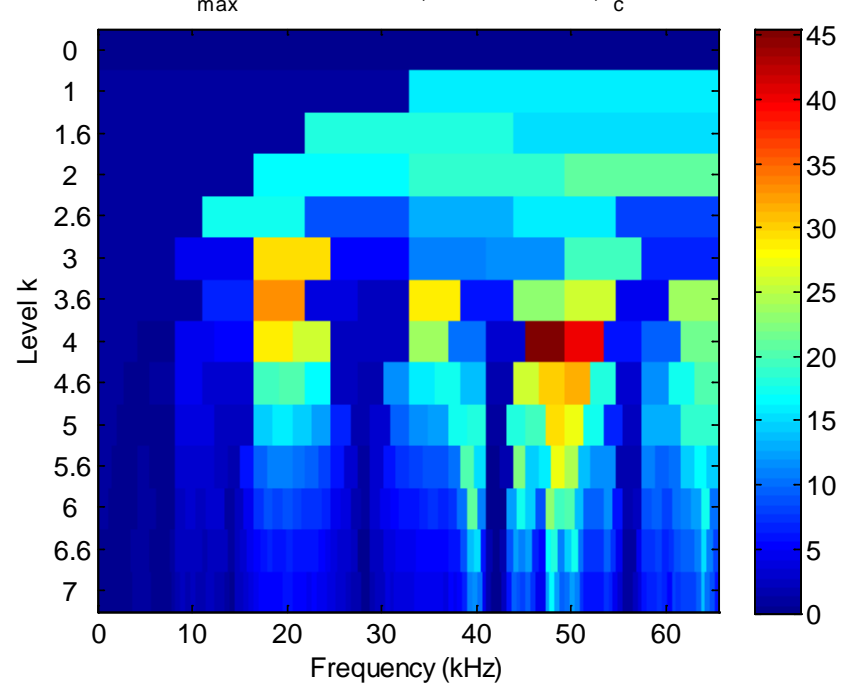

b

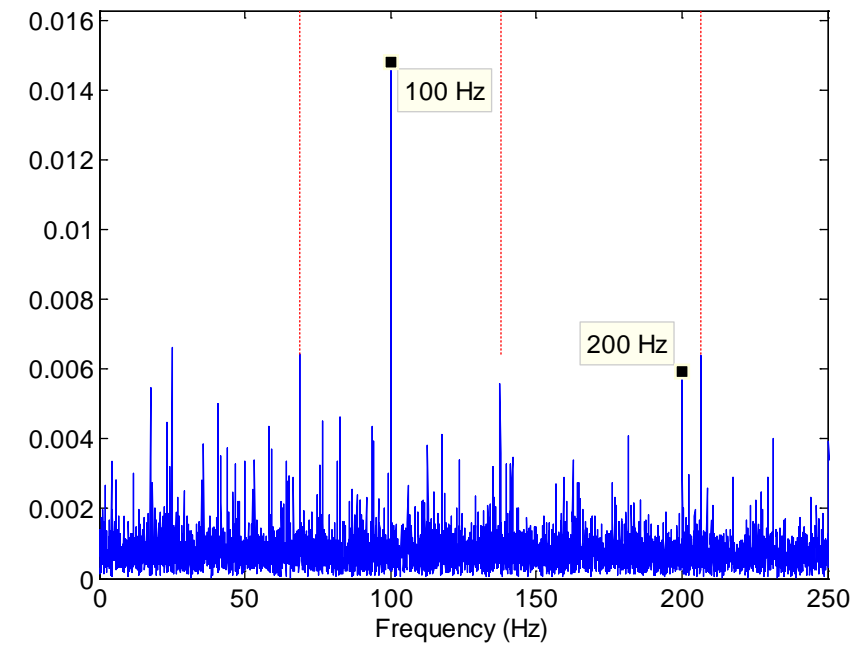

Fig. 25 Fast Kurtogram results for Case 5 (50 Nm, IRF); (a) Fast Kurtogram; (b) squared envelope spectrum (cursors at BPFI harmonics)

a

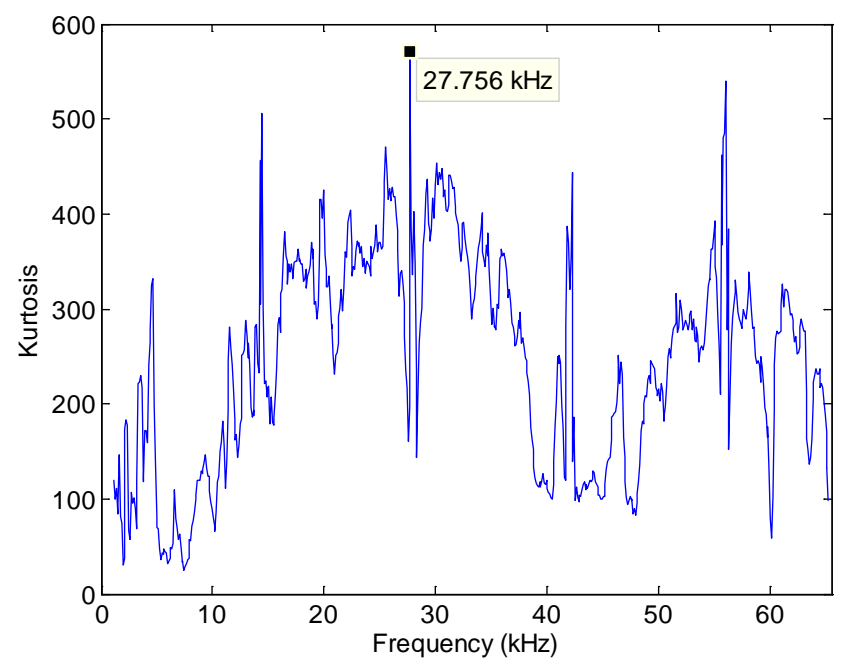

$\mathrm{b}$

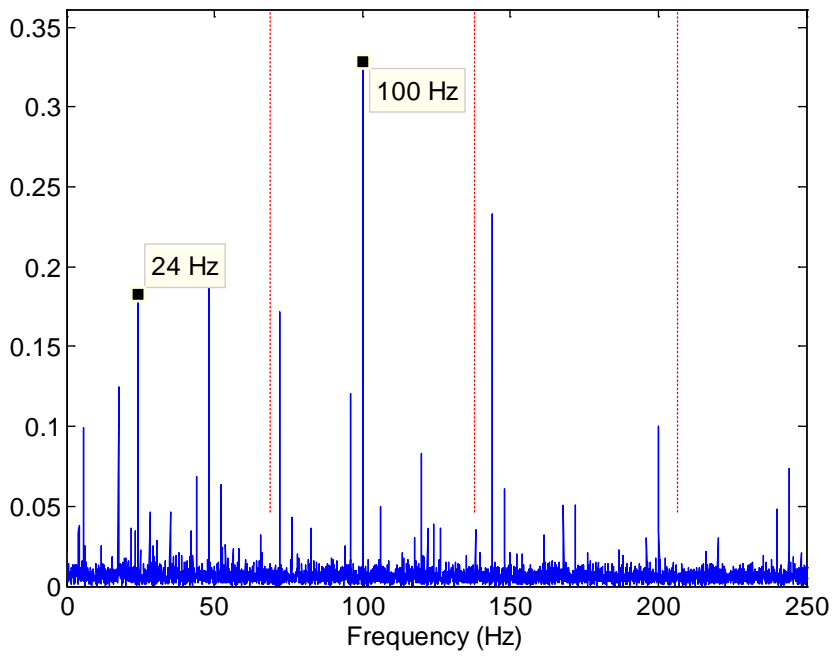

Fig. 26 Protrugram results for Case 5 (50 Nm, IRF); (a) Protrugram; (b) squared envelope spectrum (cursors at BPFI harmonics) 

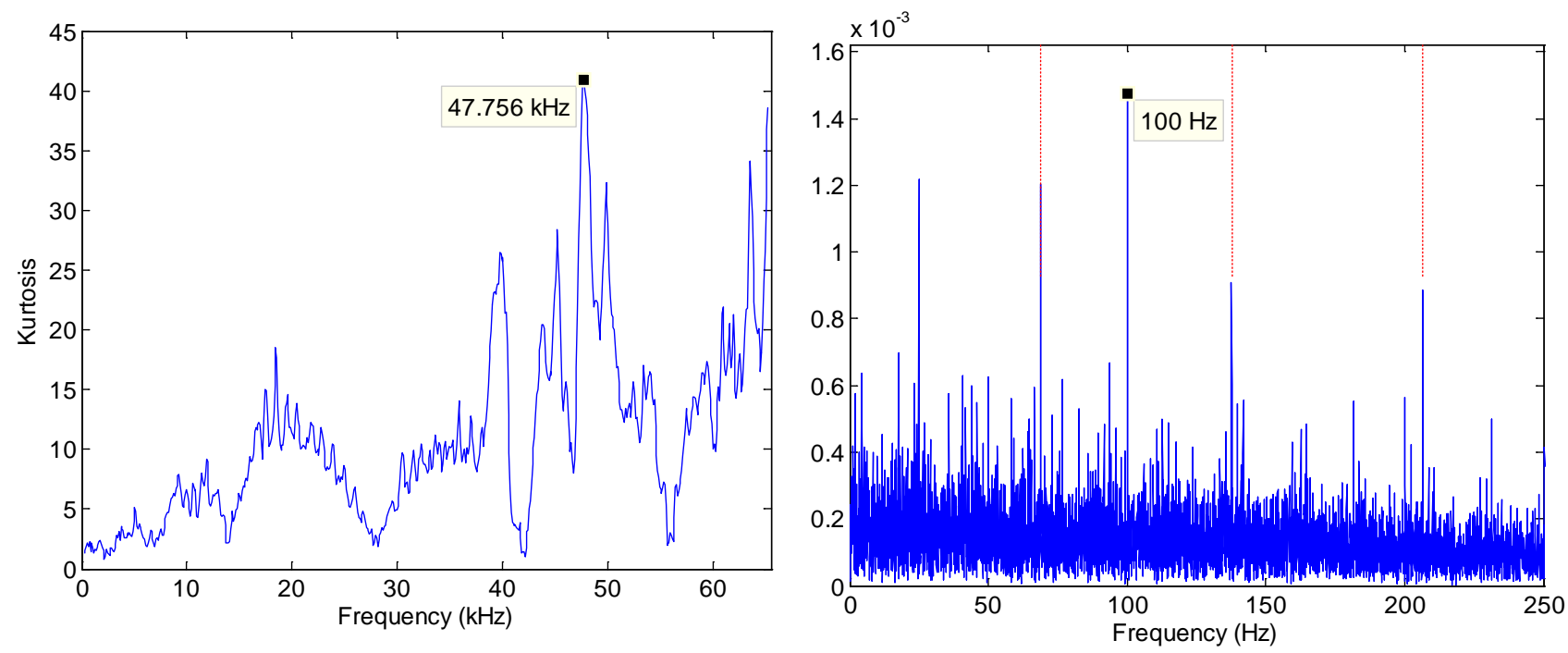

Fig. 27 Optimised SK results for Case 5 (50 Nm, IRF); (a) SK; (b) squared envelope spectrum (cursors at BPFI harmonics)

\subsection{Simulated data results}

The three methods were applied to the simulated EMI-contaminated bearing fault signal outlined in Section 4.2. The fault frequency for this signal is $69 \mathrm{~Hz}$. Again, the Fast Kurtogram was applied down to a bandwidth of $512 \mathrm{~Hz}$ (Level 7), and the Protrugram and Optimised SK were applied with a step size of $100 \mathrm{~Hz}$ and bandwidth of $512 \mathrm{~Hz}$.

The Fast Kurtogram is shown in Fig. 28(a), giving $C F=32,768 \mathrm{~Hz}$, and $B W=21,845 \mathrm{~Hz}$. The corresponding envelope spectrum is presented in Fig. 28(b), and while it does give some indication of the bearing fault, the dominant components are the even harmonics of the pseudo line frequency of $24 \mathrm{~Hz}$.

The Protrugram is shown in Fig. 29(a). The optimal $C F$ is chosen as $47,656 \mathrm{~Hz}$, again very close to one of the switching frequency $(8 \mathrm{kHz})$ harmonics. The corresponding envelope spectrum, Fig. 29(b), is dominated by the pseudo line frequency, and associated harmonics. However, as with Case 4 of the measured results (Fig. 22), in addition to the EMI-related spikes, the Protrugram shows a hump, in this case centred on the specified resonance frequency of $14 \mathrm{kHz}$. An envelope spectrum based instead on demodulating a band centred on this hump would diagnose the bearing fault. This is shown in the Optimised SK results in Fig. 30: the envelope spectrum obtained with $C F=14,456 \mathrm{~Hz}$ displays clear fault frequency harmonics. 
$\mathrm{a}$

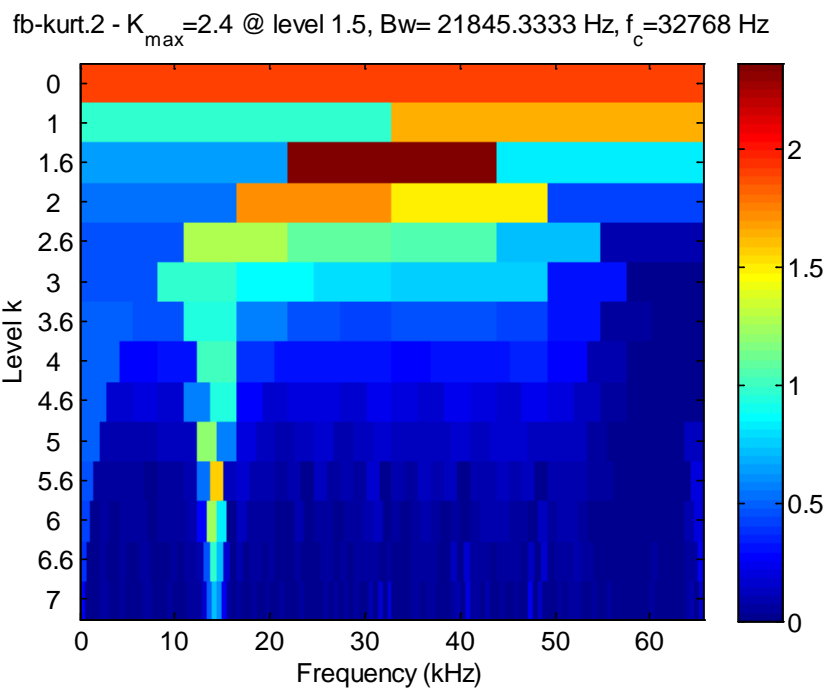

b

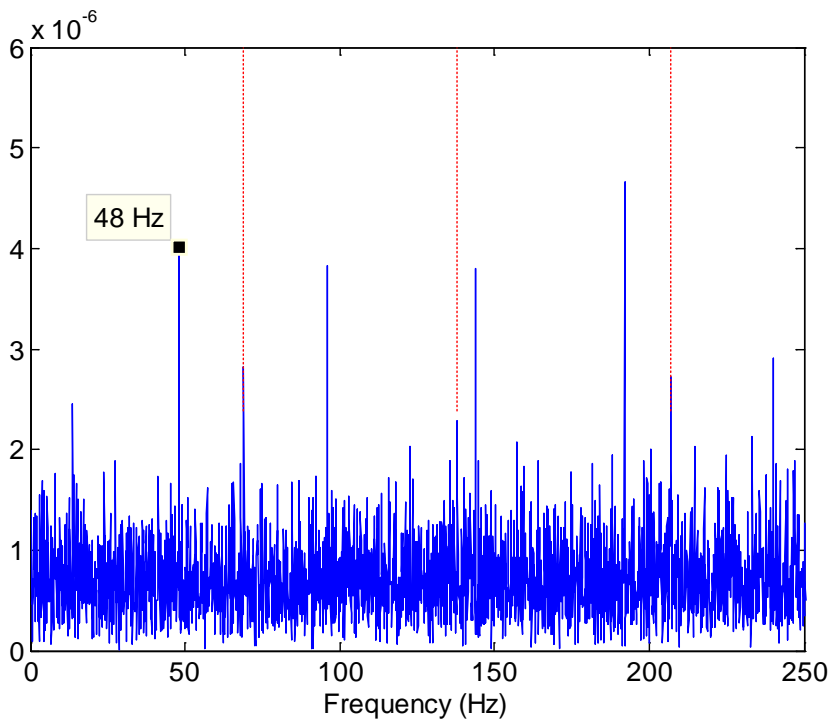

Fig. 28 Fast Kurtogram results for simulated signal; (a) Fast Kurtogram; (b) squared envelope spectrum (cursors at fault frequency harmonics)

a

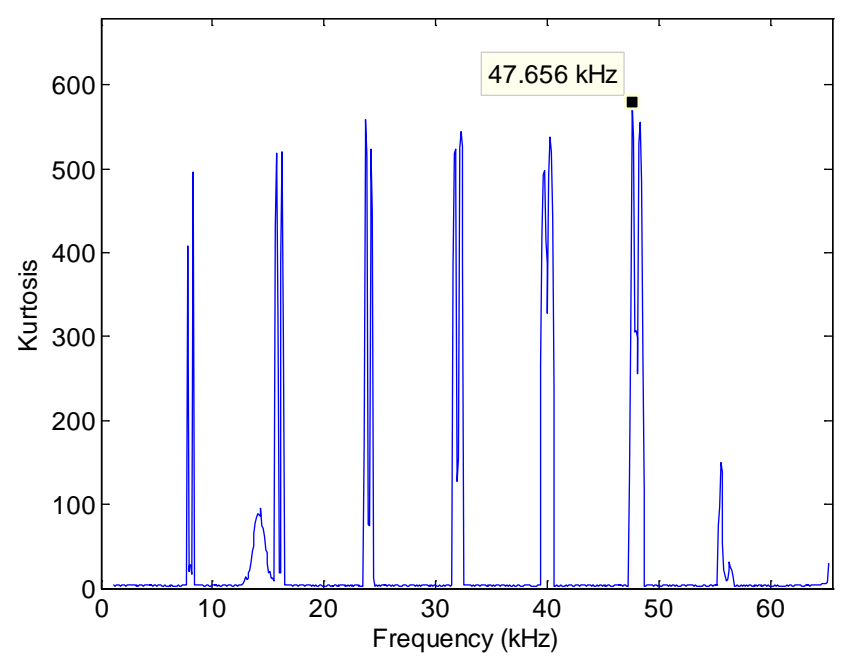

$\mathrm{b}$

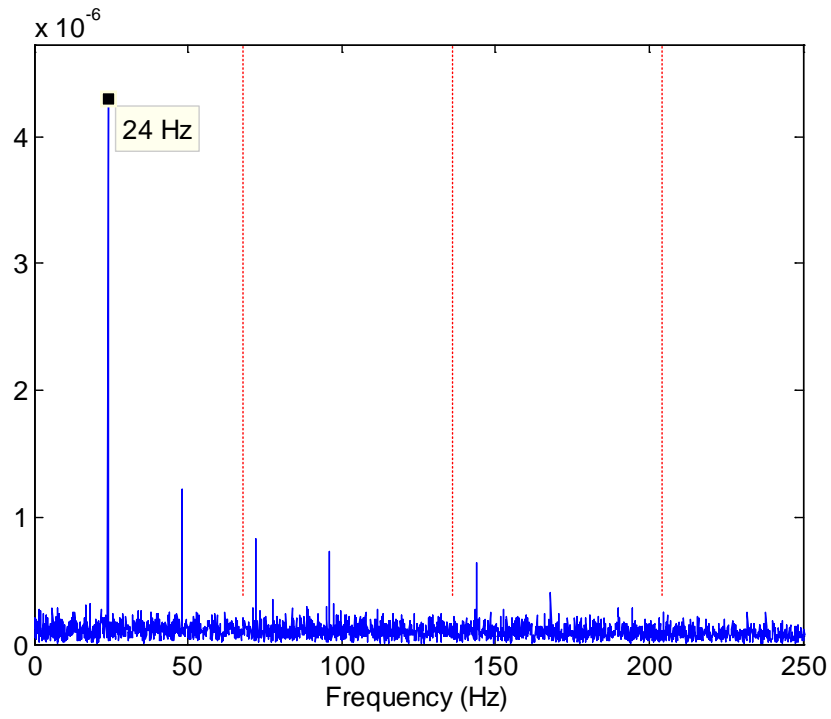

Fig. 29 Protrugram results for simulated signal; (a) Protrugram; (b) squared envelope spectrum (cursors at fault frequency harmonics) 
a

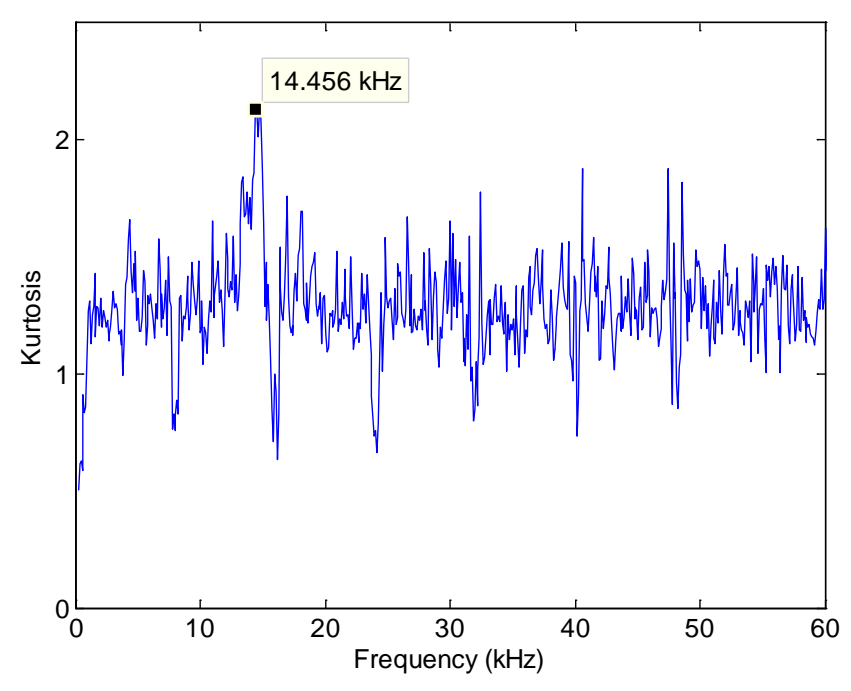

b

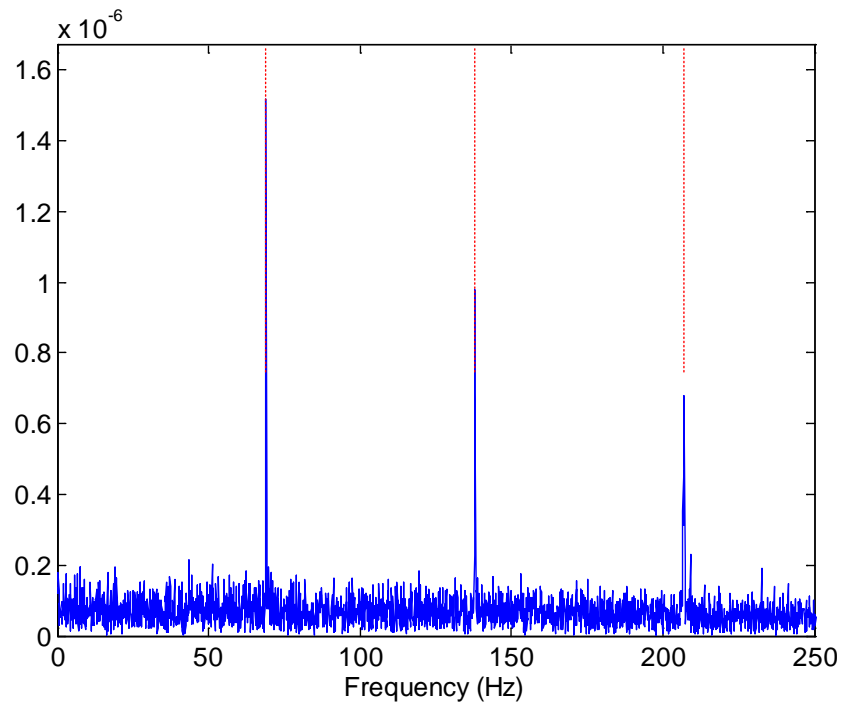

Fig. 30 Optimised SK results for simulated signal; (a) SK; (b) squared envelope spectrum (cursors at fault frequency harmonics)

\section{Discussion}

\subsection{Comparison of methods}

\subsubsection{Experimental data}

Table 2 shows a comparison of the diagnostic performance of the three investigated methods using the experimental data. Cases 1-3 represent the outer race fault with torque loads of 70, 50 and 30 Nm; Cases 4-6 are for the inner race fault with the same loads (see Table 1). The Optimised SK approach diagnosed the fault successfully in all cases, while the Fast Kurtogram was successful in half the cases (with higher loads). The Protrugram was found to be difficult to interpret in this application, proving unsuccessful in all cases when the Protrugram peak was used to select the centre frequency (see note for Case 4). The determined optimal demodulation bands are also shown in the table, and it can be seen that all successful diagnoses were based on demodulating very high frequency bands, in the range $40-65 \mathrm{kHz}$. This is perhaps due to the very small size of the bearings (with needle rollers of $2 \mathrm{~mm}$ diameter) exciting resonances only at very high frequencies. Note that the Optimised SK and Protrugram methods were applied with a centre frequency step size of $100 \mathrm{~Hz}$ and the bandwidth pre-set to $512 \mathrm{~Hz}$, while the Fast Kurtogram was applied down to a bandwidth of $512 \mathrm{~Hz}$ (Level 7). 
Table 2 Performance comparison of the Optimised SK, Fast Kurtogram and Protrugram

\begin{tabular}{ccccccc}
\hline \multirow{2}{*}{ Case } & \multicolumn{3}{c}{ Fault diagnosis successful? } & \multicolumn{3}{c}{ Selected optimal demodulation band $(\mathrm{kHz})$} \\
\cline { 2 - 7 } & Optimised SK & Fast Kurtogram & Protrugram & Optimised SK & Fast Kurtogram & Protrugram \\
\hline 1 & Yes & Yes & No & $64.4-64.9$ & $63.5-64.5$ & $14.2-14.7$ \\
2 & Yes & No & No & $40.1-40.6$ & $16.4-21.8$ & $26.0-26.5$ \\
3 & Yes & No & No & $43.9-44.4$ & $16.4-21.8$ & $55.4-55.9$ \\
4 & Yes & Yes & No* & $63.4-63.9$ & $63.5-65.5$ & $42.0-42.5$ \\
5 & Yes & Yes & No & $47.5-48.0$ & $45.1-49.2$ & $27.5-28.0$ \\
6 & Yes & No & No & $44.3-44.8$ & $16.4-21.8$ & $55.4-55.9$ \\
\hline
\end{tabular}

* The Protrugram was successful in this case when the peak of the 'hump' (rather than the spikes) was used to select the centre frequency.

It is perhaps worth reiterating here the drawbacks of the established methods in this application. The studied EMI-contaminated signals were found to have periodically impulsive (high kurtosis) interference distributed over a broad frequency range. This broadness proved problematic for the Fast Kurtogram, because it does not restrict bandwidth when blindly choosing a demodulation band, hence it tended to pass rather than filter out the interference. The periodic structure of the impulsivity in the interference caused problems for the Protrugram, whose fault indicator is very sensitive to periodic modulation, which gives an envelope spectrum represented by discrete lines at the modulation frequency and thus a very high kurtosis. These two drawbacks are overcome with the Optimised SK approach, as reflected in the results in Table 2.

\subsection{2. $\quad$ Simulated data}

For the simulated data, where the bearing fault signal was weighted to achieve an SNR (band-limited) of $0.46(-3.4 \mathrm{~dB})$, the Optimised SK was found to give a very clear diagnosis. The Fast Kurtogram was partially successful, selecting a broad demodulation band that gave some indication of the fault but was dominated by EMI effects. The Protrugram also gave an indication of the fault, reflected as a small hump at the resonance frequency excited by the fault, but this was dwarfed in the Protrugram by large spikes related to the EMI signal content.

Different SNRs were trialled to compare the methods. The Optimised SK was found to give successful diagnoses with SNRs larger than $0.43(-3.7 \mathrm{~dB})$, while the Fast Kurtogram required an SNR of $0.68(-1.7 \mathrm{~dB})$ or greater to deliver a clear diagnosis with a demodulation band centred on the resonance frequency. The Protrugram was found to be so vulnerable to the periodic modulation of the EMI that it did not give an unambiguously successful diagnosis for any of the SNR values attempted (up to $+50 \mathrm{~dB}$ ).

\subsection{Computation time}

When introducing the Protrugram, Barszcz and Jabłoński [9] discussed the trade-off between computation time and step size (which affects the potential accuracy of the $C F$ result). This same trade-off exists with the Optimised SK, yet because it is based on the envelope time signal and not the envelope spectrum, one FFT operation is saved in each loop, giving a substantial efficiency gain. The computation time as a function of step size, $s p$, for the three applied methods is shown in Fig. 
31. The figure shows processing times in MATLAB for the experimental data outlined in Section 4.1.3 (sampling rate 131,072 Hz, record length $20 \mathrm{~s}$ ). The Fast Kurtogram was applied down to a bandwidth of $512 \mathrm{~Hz}$ (Level 7) and with the parameters classical and filterbank, while a bandwidth of $512 \mathrm{~Hz}$ was used for the Protrugram and Optimised SK. Note that the computation time would be highly dependent on a number of factors (e.g., processor speed, programming language, signal length, parameter settings, etc.) and is presented here to give a rough indication only.

As expected, the figure shows that computation time is roughly proportional to $1 / s p$ for the Optimised SK and Protrugram. On average, the Optimised SK was found to give about a 25\% reduction in computation time compared with the Protrugram. The Optimised SK and Fast Kurtogram took about the same time when a step size of $10 \mathrm{~Hz}$ was used, but with a $100 \mathrm{~Hz}$ step size the Optimised SK was found to be about nine times faster than the Fast Kurtogram. This suggests a step size in the range of $10-100 \mathrm{~Hz}$ is probably optimal for many applications.

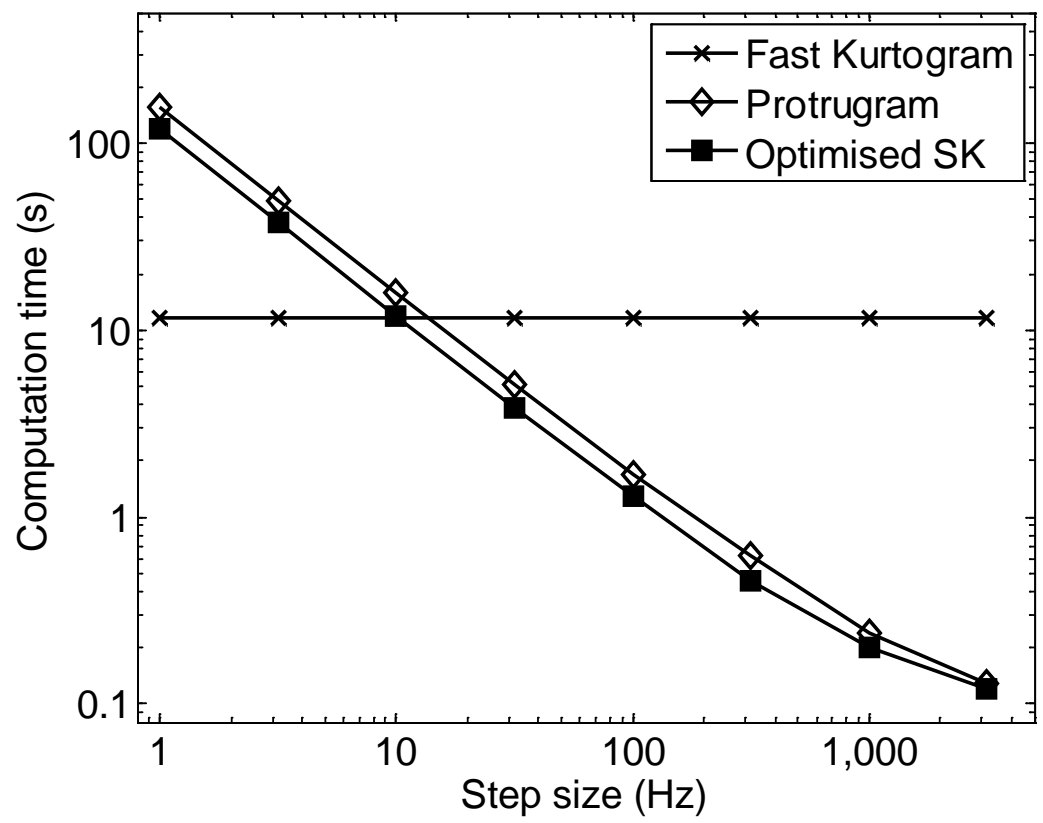

Fig. 31 Comparison of computation time vs step size for the Fast Kurtogram, Protrugram and Optimised SK; all methods applied to experimental data outlined in Section 4.1.3; Fast Kurtogram applied down to $B W=512 \mathrm{~Hz}$ (Level 7); $B W=512 \mathrm{~Hz}$ for Protrugram and Optimised SK

\subsection{Further discussion}

As with the Protrugram, the Optimised SK approach is not a blind method; the demodulation bandwidth must be set, which requires certain knowledge of the system. In this sense the approach is not as universally applicable as the Fast Kurtogram, which, of the three methods, would have the best chance of success in blind applications. It was suggested in Section 3.3 that in the presence of wideband interference, the bandwidth should be as narrow as possible, but should include at least the first three harmonics of the bearing characteristic fault frequency. On the other hand, in the presence of narrowband interference, a large bandwidth may be advantageous, and this would be very straightforward with the Optimised SK approach. 


\section{Conclusions and recommendations}

The Fast Kurtogram and Protrugram are well-established bearing diagnostics tools, but it has been shown here that they do not deal well with forms of impulsive wideband interference, in this case electromagnetic interference (EMI) from a variable frequency drive (VFD). To overcome this, the paper proposes an Optimised Spectral Kurtosis approach for dealing with EMI-contaminated signals. The Optimised SK is simply a representation of the traditional spectral kurtosis calculated using the minimum bandwidth required for the application. Unlike the Fast Kurtogram, the approach is not blind, and so knowledge of the bearing parameters is required to set the bandwidth, and the optimal centre frequency is then selected through a stepping process. The idea is that because the interference is wideband, by specifying as narrow a bandwidth as possible, the signal to noise ratio is maximised, which then allows identification of the bearing fault information.

The effectiveness of the approach was demonstrated using two data sets: EMI-contaminated vibration signals collected from a planetary gearbox test rig with seeded planet bearing faults; and, a simulated bearing fault signal containing strong EMI content in the form of large transients associated with a pulse-width modulated signal. Optimised SK was found to outperform the Fast Kurtogram and Protrugram in most cases, suggesting great potential for bearing diagnostics in applications with intense EMI or other wideband masking signals.

A further contribution of the paper is simply to bring the issue of EMI from VFDs to the attention of the condition monitoring community. In the authors' judgment, this is a more widespread problem than is commonly realised. This kind of interference generally takes the spectral form of repeated harmonic/sideband families, which remain discrete even at high frequencies. In the time domain, the interference can appear as impulsive dropouts or spikes in the signal, thus affecting traditional bearing diagnostic techniques, which tend to be based on detecting and enhancing the signal's impulsivity. In such instances, the technique outlined in this paper may prove useful, and in some cases it may be possible to alter the nature of the interference - by changing the VFD switching frequency, for example - to remove or minimise its adverse affects. In any case, it is important for analysts to be able to identify EMI content in measured vibration signals, lest it interfere with the analysis undertaken.

\section{Acknowledgements}

This research was supported in part by the Australian Research Council and SpectraQuest, through Linkage Project [LP110200738]. The authors would like to thank Andrew Bligh for the initial design of the planetary gearbox.

\section{References}

[1] R.B. Randall, J. Antoni, Rolling element bearing diagnostics - A tutorial, Mechanical Systems and Signal Processing, 25 (2011) 485-520.

[2] Y. Zhang, R.B. Randall, Rolling element bearing fault diagnosis based on the combination of genetic algorithms and fast kurtogram, Mechanical Systems and Signal Processing, 23 (2009) 1509-1517. 
[3] P.D. McFadden, J.D. Smith, Vibration monitoring of rolling element bearings by the high-frequency resonance technique — a review, Tribology International, 17 (1984) 3-10.

[4] J. Antoni, The spectral kurtosis: a useful tool for characterising non-stationary signals, Mechanical Systems and Signal Processing, 20 (2006) 282-307.

[5] J. Antoni, R.B. Randall, The spectral kurtosis: application to the vibratory surveillance and diagnostics of rotating machines, Mechanical Systems and Signal Processing, 20 (2006) 308-331.

[6] J. Antoni, Fast computation of the kurtogram for the detection of transient faults, Mechanical Systems and Signal Processing, 21 (2007) 108-124.

[7] N. Sawalhi, R. Randall, H. Endo, The enhancement of fault detection and diagnosis in rolling element bearings using minimum entropy deconvolution combined with spectral kurtosis, Mechanical Systems and Signal Processing, 21 (2007) 2616-2633.

[8] Y. Lei, J. Lin, Z. He, Y. Zi, Application of an improved kurtogram method for fault diagnosis of rolling element bearings, Mechanical Systems and Signal Processing, 25 (2011) 1738-1749.

[9] T. Barszcz, A. Jabłoński, A novel method for the optimal band selection for vibration signal demodulation and comparison with the Kurtogram, Mechanical Systems and Signal Processing, 25 (2011) 431-451.

[10] D. Wang, P.W. Tse, K.L. Tsui, An enhanced Kurtogram method for fault diagnosis of rolling element bearings, Mechanical Systems and Signal Processing, 35 (2013) 176-199.

[11] G.L. Skibinski, R.J. Kerkman, D. Schlegel, EMI emissions of modern PWM AC drives, IEEE Industry Applications Magazine, 5 (1999) 47-80.

[12] H. Zhang, Investigation and mitigation of the adverse effects of PWM adjustable speed drives, Oregon State University, PhD Thesis, 1998.

[13] K. Detrich, S. Ganeriwala, N. Sawalhi, The interference of variable frequency drives (VFDs) on the vibration signature analysis of machine defects, Tenth International Conference on Condition Monitoring and Machinery Failure Prevention Technologies, Kraków, Poland, 18-20 June, 2013.

[14] R. Lindeborg, Variable Frequency Drives, Scientific Impeller, (1998).

[15] J. Sun, Pulse-Width Modulation, in Dynamics and Control of Switched Electronic Systems, Springer London, 2012.

[16] X. Pei, K. Zhang, Y. Kang, J. Chen, Analytical estimation of common mode conducted EMI in PWM inverter, 39th IEEE Industry Applications Conference, 3-7 October, 2004, pp. 2651-2656.

[17] G. Skibinski, J. Pankau, R. Sladky, J. Campbell, Generation, control and regulation of EMI from AC drives, 32nd IEEE Industry Applications Conference, 5-9 October, 1997, pp. 1571-1583.

[18] P.E. Van Wiltenburg, Mitigation of EMI generated by a variable-frequency-drive controller for an AC induction motor, US Naval Postgraduate School, Monterey CA, Master's Thesis, 1994.

[19] J.L. Kotny, N. Idir, Time domain models of the EMI sources in the variable speed drives, IEEE Energy Conversion Congress and Exposition (ECCE), 12-16 September, 2010, pp. 1355-1360.

[20] M. Moreau, N. Idir, P. Le Moigne, Modeling of Conducted EMI in Adjustable Speed Drives, Electromagnetic Compatibility, IEEE Transactions on, 51 (2009) 665-672.

[21] D. Quezada Acuña, C. Molina Vicuña, Damage Assessment of Rolling Element Bearing Using Cyclostationary Processing of AE Signals with Electromagnetic Interference, in Cyclostationarity: Theory and Methods-II, Springer International Publishing, 2015, pp. 43-54.

[22] W. Smith, L. Deshpande, R. Randall, H. Li, Bearing diagnostics in a planetary gearbox: a study using internal and external vibration signals, 26th International Congress of Condition Monitoring 
and Diagnostic Engineering Management, Helsinki, Finland, 11-13 June, 2013.

[23] W.A. Smith, R.B. Randall, Rolling element bearing diagnostics using the Case Western Reserve University data: A benchmark study, Mechanical Systems and Signal Processing, 64-65 (2015) 100-131.

[24] J. Antoni, R. Randall, Unsupervised noise cancellation for vibration signals: part II-a novel frequency-domain algorithm, Mechanical Systems and Signal Processing, 18 (2004) 103-117.

[25] N. Sawalhi, R.B. Randall, Signal Prewhitening using Cepstrum Editing (Liftering) to Enhance Fault Detection in Rolling Element Bearings, 24th International Congress on Condition Monitoring and Diagnostic Engineering Management, Stavanger, Norway, 30 May - 1 June, 2011.

[26] R.B. Randall, N. Sawalhi, A New Method for Separating Discrete Components from a Signal, Sound and Vibration, May 2011 (2011) 6-9.

[27] W.A. Gardner, Cyclic Wiener filtering: theory and method, Communications, IEEE Transactions on, 41 (1993) 151-163.

[28] N. Sawalhi, R.B. Randall, Semi-automated bearing diagnostics - three case studies, 20th International Congress on Condition Monitoring and Diagnostic Engineering Management, Faro, Portugal, 13-15 June, 2007.

[29] P. Borghesani, P. Pennacchi, R.B. Randall, N. Sawalhi, R. Ricci, Application of cepstrum pre-whitening for the diagnosis of bearing faults under variable speed conditions, Mechanical Systems and Signal Processing, 36 (2013) 370-384.

[30] Z. Fan, H. Li, A hybrid approach for fault diagnosis of planetary bearings using an internal vibration sensor, Measurement, 64 (2015) 71-80.

[31] W. Smith, L. Deshpande, R. Randall, H. Li, Gear diagnostics in a planetary gearbox: a study using internal and external vibration signals, International Journal of Condition Monitoring, 3 (2013) 36-41.

[32] P.D. McFadden, J.D. Smith, Model for the Vibration Produced by a Single Point Defect in a Rolling Element Bearing, Journal of Sound and Vibration, 96 (1984) 69-82. 